\title{
MODELOS PARA DADOS DE PROPORÇÕES COM SUPERDISPERSÃO APLICADOS AO CONTROLE BIOLÓGICO
}

\author{
AFrÂNIO MÁrCIO CORRÊA VIEIRA \\ Estatístico
}

Orientadora: Prof ${ }^{a}$ Dr. ${ }^{a}$ CLARICE G. B. DEMÉTRIO

\begin{abstract}
Dissertação apresentada à Escola Superior de Agricultura "Luiz de Queiroz", Universidade de São Paulo, para obtenção do título de Mestre em Agronomia, Área de Concentração: Estatística e Experimentação Agronômica.
\end{abstract}

PIRACICABA

Estado de São Paulo - Brasil

Outubro - 1998 
Dados Internacionais de Catalogação na Publicação (CIP) DIVISÃo DE BIBLIOTECA E DOCUMENTAÇĀO - Campus "Luiz de Queiroz"/USP

Vieira, Afrânio Márcio Corrèa

Modelos para dados de proporções com superdispersão aplicados ao controle biológico / Afrânio Márcio Corrêa Vieira. - - Piracicaba, 1998.

$61 \mathrm{p}$.

Dissertaçāo (mestrado) - Escola Superior de Agricultura Luiz de Queiroz, 1998.

Bibliografia.

1. Análise de regressão 2. Controle biológico 3. Distribuição binomial 4. Ensaio biológico 5 . Método da máxima verossimilhança 6 . Modelo matemático 7 .

Parasitismo 8. Simulação I. Título 
"Como todos os jovens, eu decidi ser um gênio, mas felizmente o riso interveio." Clea, Lawrence Durrell 
Tive o previlégio de ter nascido em uma família especial. Se concluir um mestrado exige dedicação, disciplina e força de vontade, aprendi tudo isto com os meus pais, Afrânio e Darci Vieira e com os meus irmãos, Alfredo e Frederico Vieira. As páginas desta tese são insuficientes para que eu possa colocar em palavras o carinho e a gratidão que tenho por eles.

Eles foram também uma base, um porto seguro, um conforto nos vários momentos em que achei que a vida estava sendo injusta comigo. Foram também a melhor companhia que poderia ter nos momentos em que, com certeza, Deus me presenteava com mais um bom momento da minha vida. Obrigado ao Antônio Moreira, Cristián Carranza, Geraldo Aquino, Iran Oliveira Silva, Luciano Moreira, Marco Ferrão, Marcos Bueloni, Maria Helena Spyrides, Maurici Scarpari e Oswaldo Santana. Vocês estão presentes nas minhas orações e no meu coração.

Este meu amigo tem minha gratidão eterna, que acreditou mais em mim que eu mesmo. Ao Pedro Augusto (e ao Juca, Lolinha e Tigrinha), muitíssimo obrigado. Aonde eu for, vocês estarão comigo, sempre.

Acho que esta é mais uma etapa que completei nesta minha missão. Se cheguei aqui, contei com ajuda de vários amigos e do Grande Pai que, apesar de não estarem à nossa vista, estão sempre próximos, sempre estiveram. Obrigado. 


\section{AGRADECIMENTOS}

Este período que estive em Piracicaba foi uma vida, muito aprendi, muito cresci. Várias pessoas influenciaram direta ou indiretamente na conclusão deste trabalho. Sou eternamente grato:

- À minha orientadora Prof ${ }^{a}$ Dr $^{a}$ Clarice G. B. Demétrio e ao meu co-orientador Prof. John Hinde, da Universidade de Exeter/UK, por terem sido muito mais que orientadores; foram amigos e pais, que muito me ensinaram e não mediram esforços nos momentos em que a luz do túnel não acendia de jeito nenhum;

- Aos meus sempre mestres Prof. Dr. Enrico Colosimo (Depto. de Estatística da UFMG) e Prof. Dr. Ivan Sampaio (Depto. Zootecnia da UFMG), que apostaram em mim e agora apresento o resultado deste investimento;

- Ao Prof. Dr. José Parra e $\operatorname{Dr}^{a}$ Marta Rossi, do Depto. de Entomologia da ESALQ/USP, pelo fornecimento dos dados e pelas discussões sobre o T. galloi e os ensaios de controle biológico aplicado;

- À Prof ${ }^{a}$ Eng $^{a}$ Cristina Werkema, da Fundação de Desenvolvimento Gerencial, pelo investimento, confiança, exemplo e amizade;

- Aos professores do Departamento de Matemática e Estatística da ESALQ/USP, pelos ensinamentos que nos faz enxergar "com olhos de quem quer ver";

- Aos professores Dr. Sílvio Zocchi e $\mathrm{Dr}^{a}$ Roseli Leandro, pelo companherismo, pelas discussões, pela boa música;

- À Comissão de Aperfeiçoamento de Pessoal de Nível Superior (CAPES) pela bolsa de estudos, que sem esta, este trabalho não passaria de uma boa intenção;

- Às minhas amigas Maria Helena Spyrides e Sílvia Freitas, pela conferência nas fórmulas e demonstrações, discussões e amizade;

- Às amigas Jeanete Moreira e Sílvia Freitas, pela ajuda nas várias mudanças de casa em Piracicaba;

- À minha turma de mestrado: Adilson, Cristián, Maria Helena, Wilson, Sandra, Idemauro e os colegas do doutorado Jeanete, Paulo, Sílvia, Lêda, Denise, Cecilia, Sílvio... Minha família de Piracicaba; 
- Aos funcionários do DME, pela presteza e atenção dispensada;

- À família Alves: Vó Diva, Rosa, Lúcia e Iza, pela atenção e carinho numa fase muito importante na minha estadia em Piracicaba: o começo;

- Aos amigos de Pira, Campinas e Sampa, que fizeram do estado de São Paulo minha segunda casa: Maurici Scarpari, Fernando Pompemayer, Nilton, Nirley, Rosália, Marco e Iran, Oswaldo, Gilbert e Wagner, Marco Ferrão, Beto e Marcos, Marcelo Sotratti e Ricardo Cano. Muito Obrigado!;

- Aos amigos da Inglaterra que tornaram 2 meses da minha vida na "grande aventura": John, Kathie and Kathie's family, Stephen Powers, Richard Bullock, Andreas Frey, Nicola and Colin, Renee Menezes and friends. Thanks a lot! 


\section{SUMÁRIO}

Página

RESUMO

vi

SUMMARY

viii

1 INTRODUÇÃO 1

2 REVISÃO DA LITERATURA 3

2.1 Modelos Lineares Generalizados . . . . . . . . . . . . . . . 3

2.2 Superdispersão para Dados de Proporções . . . . . . . . . . . . . . . 4

2.2.1 Modelo para Proporções com Denominadores Iguais . . . . . . 5

2.3 Modelo Binomial Truncado . . . . . . . . . . . . . . . . . . . 6

2.4 Modelo para Mistura de Distribuições . . . . . . . . . . . . . . . . . 7

3 METODOLOGIA $\quad 9$

3.1 Material ................................ 9

3.2 Métodos . . . . . . . . . . . . . . . . . . 10

3.2 .1 Modelos Lineares Generalizados . . . . . . . . . . . . 10

3.2 .2 Modelo Binomial . . . . . . . . . . . . . . . . . . . . 12

3.2.3 Modelo Binomial Superdisperso . . . . . . . . . . . . . . 13

3.2.4 Modelo Binomial Truncado . . . . . . . . . . . . . . 16

3.2.5 Modelo Binomial Truncado Superdisperso . . . . . . . . . . 20

3.2 .6 Modelo de Mistura Bernoulli-Binomial . . . . . . . . . . . . . 20

3.2.7 Modelo de Mistura Bernoulli-Binomial Superdispersa . . . . . 23

3.3 "Half-normal Plots" . . . . . . . . . . . . . . . . . . . . . . . . 24

$4 \quad$ RESULTADOS E DISCUSSÃO $\quad 27$

$\begin{array}{lll}5 & \text { CONCLUSŌES } & 37\end{array}$

REFERÊNCIAS BIBLIOGRÁFICAS

APENDICE 1 42

$\begin{array}{ll}\text { APENDICE 2 } & 58\end{array}$ 


\title{
MODELOS PARA DADOS DE PROPORÇÕES COM SUPERDISPERSÃO APLICADOS AO CONTROLE BIOLÓGICO
}

\author{
Autor: AFRÂNIO MÁRCIO CORRÊA VIEIRA \\ Orientadora: Prof ${ }^{a}$. Dr ${ }^{a}$. CLARICE G. B. DEMÉTRIO
}

\section{RESUMO}

O Controle Biológico de Pragas é um importante ramo da Entomologia, fornecendo formas ambientalmente corretas de controle de insetos. Bioensaios são utilizados para encontrar condições ótimas para a produção de parasitas e estratégias para aplicação no campo. Em alguns destes ensaios, proporções são medidas e, frequentemente, estes dados possuem um número excessivo de zeros. Neste trabalho, seis modelos serão aplicados a um conjunto de dados obtido de ensaios de controle biológico para Diatraea saccharalis, uma praga comum em plantações de Cana-deAçúcar.

Uma escolha natural para modelagem de dados de proporções é o modelo binomial. O segundo modelo será uma versão superdispersa do modelo binomial, estimado pelo método da quase-verossimilhança estendida. Este modelo foi construído inicialmente para modelar superdispersão gerada pela variabilidade individual na probabilidade de sucesso. Quando o interesse maior é sobre os valores positivos das proporções, um modelo pode ser baseado na distribuição binomial truncada, assim como na sua versão superdispersa. Os dois últimos modelos incluem os zeros e são baseados em um modelo de mistura finita de distribuições que utilizam a distribuição binomial e uma de suas versões superdispersas, para os dados positivos truncados.

Os seis modelos foram comparados através do uso do "half-normal plot" com envelopes de simulação, uma ferramenta gráfica que auxilia na avaliação da qualidade de ajuste dos modelos. $O$ modelo binomial truncado superdisperso mostrou-se adequado para modelar a proporção de ovos de Anagasta kuehniella que 
foram parasitados por Trichogramma galloi. Assumindo esta distribuição probabilística, uma regressão polinomial de grau cúbico foi ajustada, utilizando polinômios ortogonais, para as duas linhagens estudadas de T. galloi. Estes modelos permitiram estimar o número máximo de ovos parasitados, para cada linhagem, o que permite ao entomologista utilizar um número de fêmeas que otimiza o número de ovos parasitados e que economiza recursos do laboratório, tempo e dinheiro. 


\title{
OVERDISPERSED PROPORTION DATA MODELS APPLIED TO BIOLOGICAL CONTROL
}

\author{
Author: AFRÂNIO MÁRCIO CORRÊA VIEIRA \\ Adviser: Prof ${ }^{a}$. Dr ${ }^{a}$. CLARICE G. B. DEMÉTRIO
}

\section{SUMMARY}

Biological Control of pests is an important branch of entomology, providing environmentally friendly forms of crop protection. Bioassays are used to find the optimal conditions for the production of parasites and strategies for application in the field. In some of these assays, proportions are measured and, often, these data have an inflated number of zeros. In this work six models will be applied to datasets, obtained from biological control assays for Diatraea saccharalis, a common pest in sugar cane production. A natural choice for modelling proportion data is the binomial model. The second model will be an overdispersed version of the binomial model, estimated by an extended quasi-likelihood method. This model was initially built to model overdispersion generated by individual variability in the probability of success. When interest is only in the positive proportion data, a model can be based on the truncated binomial distribution and in its overdispersed version. The last two models include the zero proportions and they are based on a finite mixture model, where are used the binomial distribution and one of its overdispersed version for the positive data.

The six models was compared through the use of "half-normal plot" with simulated envelopes, a graphic tool that helps to assess the goodness-of-fit of the models. The overdispersed truncated binomial model was the best model to modelling the proportion of eggs of Anagasta kuehniella, that was parasitised by Trichogramma galloi. Assuming this probabilistic distribution, an order-three polynomial regression model was fitted, using orthogonal polynomials, for two species of T. galloi. These models able us to estimate the maximum number of parasitised eggs, for each species, allowing the entomologists to use the optimal number of females, saving lab resources, money and time. 


\section{INTRODUÇÃO}

O manejo de pragas através do Controle Biológico Aplicado (CBA) é uma realidade em todo o mundo. Esta técnica consiste em utilizar predadores e/ou parasitas potenciais para o controle de pragas que ocorrem com frequência na Agricultura. Segundo Parra e Zucchi (1997), esta área teve um grande avanço após a década de 60 , com o desenvolvimento de técnicas de criação de insetos de forma intensiva. No Brasil as pesquisas voltadas para o CBA ganharam impulso com o treinamento de técnicos e com a criação da pós-graduação em Entomologia. Especificamente no Brasil, uma praga que vem sendo combatida através de CBA é a Broca-da-cana (Diatraea saccharalis Fabricius, 1794). D. saccharalis é a principal praga da cana-de-açúcar no Brasil, que gerou prejuízos estimados em US $\$ 119$ milhões em 1983, somente no estado de São Paulo (Botelho, 1985).

Com a constatação de Botelho (1985) de que a fase do ovo é o fatorchave para o controle biológico de $D$. saccharalis, torna-se interessante fazer este controle através de parasitóides de ovos, ao invés de se utilizarem parasitóides larvais ou predadores do inseto adulto. A utilização de parasitóides do gênero Trichogramma, conhecidos por serem parasitas de ovos de insetos da ordem Lepdoptera, tem sido uma fonte para as pesquisas do Laboratório de Biologia, do Departamento de Entomologia da ESALQ/USP. A espécie T. galloi (Trichogramma galloi Zucchi, 1988) é um parasitóide específico de D. saccharalis (hospedeiro natural). Uma de suas características é sua baixa capacidade de parasitar ovos do hospedeiro alternativo A. kuenhiella (Anagasta kuehniella Zeller, 1979) em condições de laboratório. O interesse principal é produzir um grande número de ovos de $A$. kuehniella, parasitados pelo T. galloi e que possam ser utilizados em liberações inundativas no campo (Parra, 1997). Como o custo de criação de A. kuehniella é menor em relação à criação de $D$. saccharalis (levando-se em conta a infra-estrutura, tempo e material), torna-se interessante conhecer o processo de competição entre fêmeas de T. galloi sob o hospedeiro alternativo.

Em ensaios entomológicos, onde se tem interesse no estudo da resposta 
funcional do parasita ou do processo de competição entre fêmeas, são gerados dados na forma de proporções e/ou contagens. Frequentemente, estes dados apresentam uma variabilidade maior do que aquela que os modelos probabilísticos para proporções e contagens poderiam acomodar (ou modelar). Este fenômeno é conhecido como Superdispersão e, como consequência, erros padrões para os efeitos de fatores e covariáveis estarão subestimados. Além disso, a probabilidade do erro tipo I, associada a testes de hipóteses dos parâmetros estimados pelo modelo, estará inflacionada, e portanto, levando a conclusões erradas. Assim, é interessante e prudente utilizar modelos estatísticos que levem em consideração a superdispersão presente nos dados. Após considerar um modelo adequado à superdispersão, é de interesse do pesquisador obter o máximo de informações referentes aos processos de competição dos insetos.

O presente trabalho tem como objetivos:

1. Modelar os dados de um ensaio de controle biológico onde foi utilizado o $T$. galloi;

2. Comparar modelos probabilísticos alternativos ao modelo binomial, no processo de modelagem da superdispersão, para dados de proporções inflacionados de zeros e desenvolver os programas computacionais necessários;

3. Utilizar métodos gráficos de diagnósticos para avaliar a qualidade de ajuste dos modelos;

4. Sugerir o número ideal de fêmeas do parasita que maximize o número de ovos parasitados. 


\section{REVISÃO DA LITERATURA}

Algumas metodologias para modelagem de dados binomiais e de contagem foram propostas antes da década de 70 (Finney, 1947; Grizzle et al., 1969) mas o interesse de se unificar os procedimentos de modelagem era eminente. Já se sabia, naquela época, que dados com distribuição Binomial, Poisson, Normal, Gama e outras, tinham algo em comum em termos de probabilidade e inferência estatística: pertenciam à Familia Exponencial (Bickel \& Doksum, 1977). Entretanto, a unificação do procedimento de modelagem, sob o nome de Modelos Lineares Generalizados, foi proposta por Nelder \& Wedderburn (1972) e, através da Análise de "Deviance" ${ }^{1}$, generaliza a Análise de Variância para dados com distribuição normal e incorpora os modelos log-lineares para tabelas de contingência, análise probit, regressão logística e regressão Poisson, ligando a análise de dados discretos e contínuos. A metodologia dos Modelos Lineares Generalizados (MLG) vem ganhando cada vez mais espaço na literatura estatística e entre os estatísticos, já que esta mostra-se conceitual e computacionalmente simples e geral. Este enfoque de modelagem de dados permite que modelos mais genéricos sejam utilizados, como aqueles para dados longitudinais e para dados com superdispersão.

\subsection{Modelos Lineares Generalizados}

Uma vasta literatura sobre Modelos Lineares Generalizados está disponivel. McCullagh \& Nelder (1989) é considerada a principal referência do assunto, apesar de ser difícil para uma primeira leitura. Cordeiro (1986) e Demétrio (1993) são as referências em português, onde os detalhes do processo de estimação estão desenvolvidos e o uso do GLIM é enfatizado. Dobson (1990) é uma introdução aos modelos lineares generalizados, onde as demonstrações rigorosas cedem espaço para aplicações de modelos log-lineares, regressão logística e também regressão múltipla e ANOVA. Healy (1988) é uma introdução bastante prática ao

\footnotetext{
${ }^{1}$ Esta é uma expressão inglesa traduzida como "Desvio" por Cordeiro (1986).
} 
GLIM e Francis et al. (1993) é a referência para quem deseja utilizar e conhecer profundamente a linguagem de programação do GLIM. Aitkin et al. (1989) tratam de uma descrição prática e detalhada do uso do GLIM em situações reais envolvendo modelos para dados normais, binomiais, multinomiais, de contagem e sobrevivência.

Análise de dados provenientes de ensaios do tipo dose-resposta são bastante frequentes em estudos teratológicos, farmacológicos e em entomologia. Morgan (1992) trata deste tipo de dados (resposta binária e regressor contínuo) e questões como tempo até uma resposta, superdispersão, métodos robustos e planejamento de experimentos para este tipo de ensaio. Para análise de dados categorizados (nominais ou ordinais) no enfoque MLG a referência é Agresti (1990).

Collett (1991) é o texto ideal para quem deseja estudar e utilizar modelos para dados binários e de proporções no enfoque MLG. Sua abordagem prática e, ao mesmo tempo, didática e consistente, permite a um público maior o entendimento da metodologia. Temas específicos da Bioestatística como estudos coorte e caso-controle, bioensaios, ensaios pareados, ensaios de dose-resposta e estudos epidemiológicos são abordados. A construção do modelo linear generalizado, a descrição da função de verossimilhança, o algoritmo de estimação, qualidade do ajuste do modelo e interpretação dos dados são didaticamente bem detalhados. No capítulo de bioensaios o tema Resposta Natural é abordado para tratar de dados onde, por exemplo, insetos morrem sem terem sido submetidos a um tratamento específico. Isto leva a considerar que uma parte da população de insetos naturalmente responde a um tratamento nulo com placebo e a outra parte irá responder somente sob o tratamento em questão. Este conceito será a base dos modelos de mistura de distribuições que serão tratados na seção de Metodologia. Além do tratamento completo do assunto de diagnósticos e dos softwares para análise de respostas binárias, o tema Superdispersão recebe uma atenção especial. As causas potenciais que geram superdispersão iniciam o capítulo, seguidas de como modelar a variação entre as probabilidades da resposta, como modelar a correlação entre as respostas binárias e o caso particular de denominadores iguais para as proporções. O modelo beta-binomial e o modelo de efeito aleatório também são tratados como alternativas para dados binomiais com superdispersão.

\subsection{Superdispersão para Dados de Proporções}

Williams (1982) propõe uma maneira de se modificar o algoritmo de estimação de um modelo de regressão logística, para incorporar o efeito de superdis- 
persão para dados de proporções (ou variação extra-binomial). Este método consiste em se fazer uma sequência de ajustes do modelo binomial com pesos $1 /\left[1+\tilde{\phi}\left(m_{i}-1\right)\right]$, onde $\tilde{\phi}$ é a estimativa do parâmetro de superdispersão e $m_{i}$ é o denominador da proporção da i-ésima observação. Após algumas iterações do algoritmo, alternando a estimação do vetor de parâmetros $\beta$ e do parâmetro de superdispersão $\phi$, a convergência é obtida e assim torna possível corrigir os erros padrões de $\beta$ utilizando a estimativa final de $\phi$. Hinde (1996) apresenta uma modificação da macro para o programa GLIM, inicialmente apresentada em Collett (1991), onde o procedimento de Williams está implementado. Nesta nova versão é permitida a utilização de outras funções de ligação, além da canônica. Para verificação da qualidade do ajuste do modelo, Demétrio \& Hinde (1997) propōem a utilização do "half-normal plot" com envelopes de simulação. sendo este um critério gráfico e de fácil interpretação.

Hinde \& Demétrio (1998) apresentam uma revisão de métodos de estimação de modelos superdispersos para dados de proporções e de contagens. Eles descriminam os modelos para superdispersão em duas categorias:

- modelos que assumem a função de variância em uma forma geral, onde esta pode ter parâmetros adicionais. Em geral é utilizado o método da quaseverossimilhança (Wedderburn, 1974) para estimação dos parâmetros do modelo e métodos como o de Newton-Raphson para a estimação dos parâmetros adicionais;

- modelos probabilísticos em dois estágios para a resposta. Isto significa assumir uma distribuição probabilística para o parâmetro do modelo a ser estimado. Utilizando-se distribuiçöes conjugadas é possível obter estimativas de máxima verossimilhança, apesar de que, em geral, métodos aproximados são utilizados.

Métodos de estimação como máxima verossimilhança, momentos, quaseverossimilhança estendida, pseudo-verossimilhança e máxima verossimilhança nãoparamétrica são apresentados e suas relações são exploradas.

\subsubsection{Modelo para Proporçōes com Denominadores Iguais}

Segundo Collett (1991), a função de variância para o modelo descrito em Williams (1982),

$$
\operatorname{Var}\left(y_{i}\right)=m_{i} \pi_{i}\left(1-\pi_{i}\right)\left[1+\left(m_{i}-1\right) \phi\right]
$$


fica simplificada quando os denominadores das proporções são fixos (seja $m$ ), de onde se tem

$$
\begin{aligned}
\operatorname{Var}\left(y_{i}\right) & =m \pi(1-\pi)[1+(m-1) \phi] \\
& =m \pi(1-\pi) \phi .
\end{aligned}
$$

Este modelo é denominado Modelo com Fator de Heterogeneidade ou Modelo com Superdispersão Constante. A estimativa do parâmetro de superdispersão é obtido de forma não iterativa, através de $X^{2} /(n-p)$, onde $X^{2}$ é a estimativa da estatística de Pearson ajustada para um modelo completo, com $n$ observações e $p$ parâmetros. Uma argumentação mais técnica sobre este resultado está descrito em McCullagh \& Nelder (1989).

\subsection{Modelo Binomial Truncado}

Se for considerado que proporções iguais a zero não são por si só informativas e que o interesse maior é sobre as proporções onde pelo menos um ovo foi parasitado, pode-se pensar em um modelo probabilístico que exclua valores nulos, assumindo somente os valores $1,2, \ldots, m_{i}$, onde $m_{i}$ é o denominador da proporção $y_{i} / m_{i}$. Johnson \& Kotz (1962) apresentam a distribuição binomial truncada para o valor zero, assim como as expressões da esperança e variância. Pode-se demonstrar que esta distribuição probabilística pertence à família exponencial de distribuições e, portanto, esta pode ser utilizada no contexto MLG como distribuição de uma variável resposta.

Ridout \& Demétrio (1992) descrevem um procedimento de como utilizar a distribuição Poisson positiva (ou Poisson truncada) no enfoque MLG apresentando, também, uma macro para o GLIM. Este procedimento será utilizado para se criar um Modelo Binomial Truncado que será também implementado para o programa GLIM. Fioravante (1995) utiliza o modelo Poisson truncado, conforme descrito em Ridout \& Demétrio (1992), na modelagem de dados de contagem obtidos de experimentos de cultura de tecidos.

Thomas \& Gart (1971) fazem um estudo da performance de alguns estimadores para o parâmetro $\pi$ da distribuição binomial truncada, sob pequenas amostras. Neste artigo vê-se que para pequenas amostras (ou denominador pequeno), o estimador de máxima verossimilhança de $\pi$ tende a apresentar um vício 
considerável, principalmente com $m_{i} \leq 8$. Esta dependência da grandeza do denominador da proporção (que não ocorre no caso da Poisson truncada) deve ser levada em conta no processo de estimação para a obtenção de estimativas confiáveis.

\subsection{Modelo para Mistura de Distribuiçōes}

Uma abordagem do problema de dados de proporções com excesso de zeros, sob outro ponto de vista, é pensar sobre a incapacidade natural de algumas fêmeas em parasitar os ovos do hospedeiro alternativo. Assim, seria razoável pensar que uma parte das fêmeas não parasita e que a outra parte parasita. Assumindo que $\alpha$ é a probabilidade de uma fêmea parasitar e $1-\alpha$ a probabilidade de uma fêmea não parasitar, pode-se pensar que $Y$, o número de ovos parasitados, tenha a forma

$$
\left\{\begin{aligned}
Y & \equiv 0 \text { com probabilidade } 1-\alpha ; \\
Y & \sim P(Y=y) \text { com probabilidade } \alpha,
\end{aligned}\right.
$$

onde $P(Y=y)$ é uma distribuição probabilística adequada. Modelos sob este tipo de distribuição são conhecidos como Modelos com Mistura de Distribuições. Algumas referências da literatura tratam deste tipo de modelos, como os textos de Morgan (1992), Collett (1991), Brooks et al. (1997), Lambert (1992) e Zocchi (1993).

Collett (1991) e Morgan (1992) abordam o uso de modelos de mistura para dados de mortalidade e imunidade natural. Morgan (1992) detalha basicamente o uso de misturas de distribuições para dados de ensaios dose-resposta, descrevendo as expressões de verossimilhança e o processo de estimação dos parâmetros desconpecidos. O Algoritmo EM é sugerido como alternativa a outros algoritmos como os de Newton-Raphson e o Simplex. O algoritmo EM foi proposto por Dempster et al. (1977) e tornou se bastante popular entre os estatísticos devido à sua flexibilidade e generalidade. Este algoritmo tem sido bastante utilizado na modelagem de dados incompletos, dados agrupados onde se desconhece o número de grupos, imputação de dados em esquemas de amostragem complexos e, em especial, para mistura de distribuições.

Zocchi (1993) utiliza modelos de mistura probit, logit e complemento log-log para modelar dados de um ensaio dose-resposta com moscas (Musca domestica). Brooks et al. (1997) utilizam diversos modelos de mistura para 6 conjuntos de dados de ensaios biológicos com camundongos. Alternativamente, é utilizada neste trabalho, a estimação por máxima verossimilhança perfilada para se estimar o parâmetro de mistura $\alpha$. 
Em Lambert (1992) é proposto um modelo de mistura de distribuições Bernoulli-Poisson para se modelar o excesso de zeros na contagem de defeitos de fabricação de placas de circuito impresso. Esta metodologia, aplicada a dados de contagem, mostra-se de grande generalidade devido ao fato de ser possível associar regressores que influenciem no parâmetro de mistura $\alpha$. A metodologia proposta também permite a utilização de pacotes estatísticos como o $\mathrm{S}+$ e o GLIM para sua implementação. 


\section{METODOLOGIA}

\subsection{Material}

Os dados a serem utilizados são provenientes de um experimento realizado no Laboratório de Biologia do Departamento de Entomologia da ESALQ/USP, pela Drâ Marta Rossi, sob a coordenação do Dr. José Roberto P. Parra. Foram utilizadas nos ensaios, duas limhagens de T. galloi. A primeira foi coletada parasitando naturalmente ovos de $D$. saccharalis e mantida no Laboratório de Biologia por um número desconhecido de gerações, parasitando o hospedeiro alternativo $A$. kuehniella, o que permitiu sua perfeita adaptação (Linhagem AA). A segunda linhagem foi cedida pela COPERSUCAR (Unidade Experimental de Piracicaba), onde era mantida no hospedeiro natural D. saccharalis (Linhagem DA).

$\mathrm{O}$ interesse principal dos entomologistas, neste tipo de experimento, é identificar as condições ideais para a produção de um grande número de ovos parasitados, que serão utilizados em liberações inundativas no campo.

$\mathrm{O}$ experimento consistiu em conhecer o processo de competição de fêmeas de T. galloi (ambas linhagens), variando o número de fêmeas $(2,4,8,16$, 32,64 e 128) para parasitar um número fixo de ovos do hospedeiro alternativo (128 ovos).

Os ensaios foram conduzidos em estufas sob a temperatura de $25 \pm 1^{\circ} \mathrm{C}$, umidade relativa do ar de $70 \pm 10 \%$ e fotofase de 16:8 (16 horas com luz, 8 horas na penumbra).

As fêmeas do parasita com menos de 24 horas de idade, foram isoladas em tubos de vidro de $1 \mathrm{~cm}$ de diâmetro por $7 \mathrm{~cm}$ de comprimento, tampados com algodão, tendo sido os parasitóides alimentados com mel puro. Para cada condição experimental (número de fêmeas e número de ovos a serem parasitados) foram consideradas 10 repetições. Os ovos do hospedeiro alternativo A. kuehniella útilizados, tinham menos de 24 horas de idade e foram esterilizados em lâmpada germicida por 45 minutos. O tempo de parasitismo foi de 24 horas a $25^{\circ} \mathrm{C}$, eliminando-se os para- 
sitóides em seguida. Após um período de 6 dias a $25^{\circ} \mathrm{C}$, foi contado o número de ovos de A. kuehniella escurecidos (indicativo de que o ovo hospedeiro foi parasitado) em relação ao número inicial de ovos. O interesse dos entomologistas é saber os efeitos de linhagens e número de fêmeas sobre a proporção de ovos parasitados, e especificamente, qual o número ideal de fêmeas para que se obtenha uma maior proporção de ovos parasitados. Na Figura 1 são apresentados os diagramas de dispersão para as 2 linhagens estudadas.

No diagrama referente à linhagem DA, vê-se a ocorrência excessiva de proporçôes iguais a zero, o que gera uma possível superdispersão e que inabilita a utilização de modelos que não tratam desta variação excessiva, em termos de inferências sobre os parâmetros.

Após a execução do experimento foi obtido o número de ovos parasitados, em relação ao número inicial de ovos $(m)$, para cada condição experimental (número de fêmeas). Portanto, pode-se considerar a variável resposta $Y$ como sendo "o número de ovos parasitados dentre $m$ ovos", onde $m=128$ ovos. Os dados estão apresentados no Apêndice 2.

\subsection{Métodos}

Em um contexto geral, considere a variável aleatória $Y, y_{i}$ uma realização desta variável e $y_{i} / m_{i}$ representando a proporção de ovos parasitados da $i$-ésima observação, $i=1, \ldots, n$, sendo $n$ o tamanho da amostra. Os modelos utilizados neste trabalho são seis, descritos nas seções seguintes.

\subsubsection{Modelos Lineares Generalizados}

Os modelos abordados neste trabalho são ou utilizam modelos lineares generalizados. Como este é um assunto já bastante explorado, conforme descrito na Revisão da Literatura, será apresentada aqui apenas, em termos gerais, a definição destes modelos. Uma descrição mais completa pode ser encontrada em McCullagh \& Nelder (1989), Dobson (1990) e Demétrio (1993).

Considerando uma amostra aleatória de tamanho $n\left(y_{i}, \mathbf{x}_{i}\right)$, onde $\mathbf{x}_{i}=$ $\left(x_{1 i}, x_{2 i}, \ldots, x_{p i}\right)^{T}$ é um vetoi coluña de $(p-1)$ variáveis explanatórias, um modelo linear generalizando é definido por três componentes :

- Variáveis aleatórias dependentes $Y_{i}, i=1, \ldots, n$, pertencentes á Familia Exponencial de Distribuições, com médias $\mu_{i}$ e parâmetro de escala constante $\phi$, 

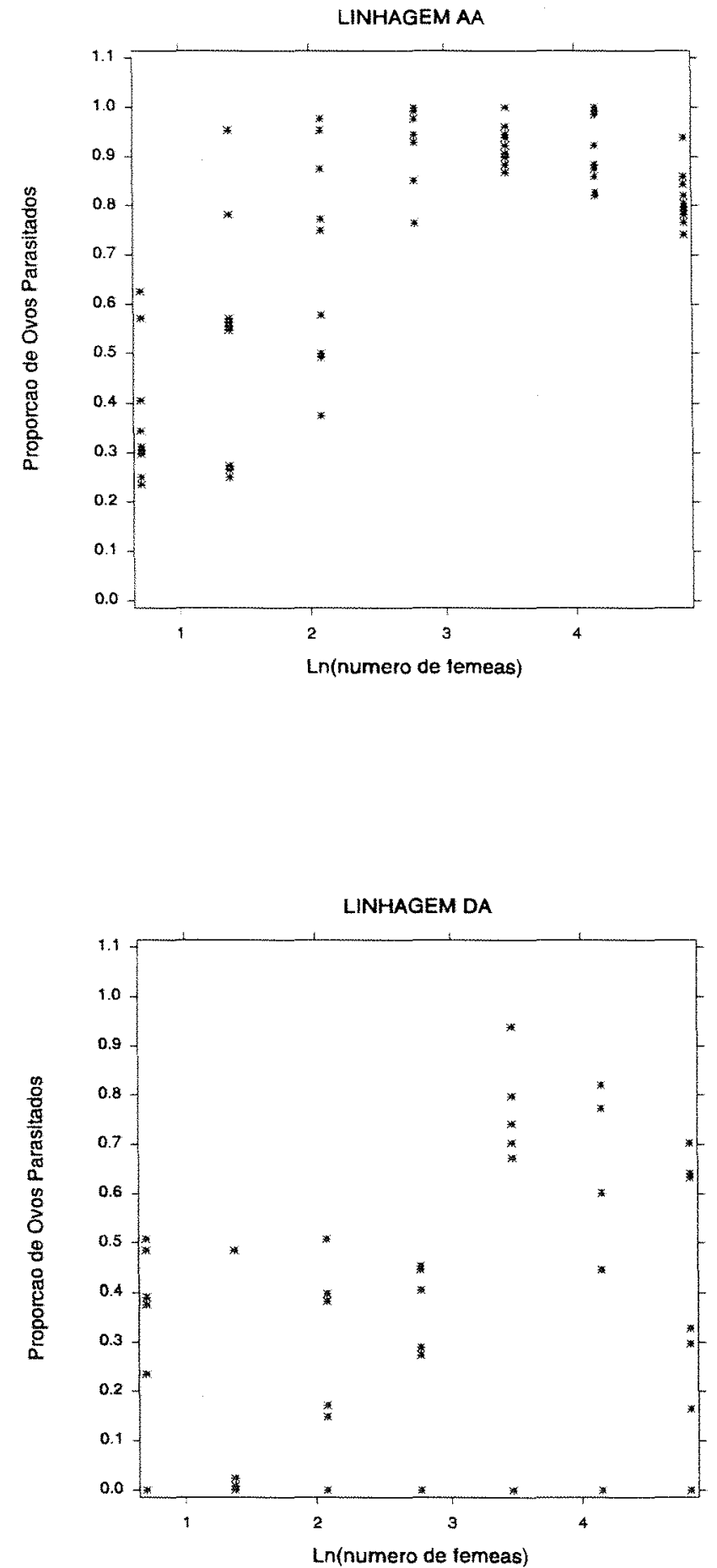

Figura 1: Diagramas de dispersão da proporção de ovos parasitados versus número de fêmeas, para as linhagens AA e DA. 
ou seja, observaçōes com densidade da forma

$$
f(y)=\exp \left\{\frac{y \theta-b(\theta)}{\phi}+c(y, \phi)\right\}
$$

onde $\mu=E[Y]=b^{\prime}(\theta)$ e $\operatorname{Var}(Y)=\phi b^{\prime \prime}(\theta)$, com $\theta$ denominado parâmetro natural ou canônico;

- O preditor linear $\eta$ dado por

$$
\eta=X \beta
$$

onde $\boldsymbol{\beta}$ é um vetor de $p$ parâmetros e $\mathbf{X}=\left[\mathbf{x}_{1}, \mathbf{x}_{2}, \ldots, \mathbf{x}_{n}\right]^{T}$ é uma matriz de delineamento de dimensões $n \times p$;

- Uma função ligação $g(\cdot)$, inversível e diferenciável, relacionando a média da distribuição ao preditor linear, ou seja,

$$
g\left(\mu_{i}\right)=\eta_{i}=\mathbf{x}_{i}^{T} \beta
$$

\subsubsection{Modelo Binomial}

Assumindo que $Y$ tem distribuição binomial, um modelo linear generalizado pode ser especificado por

$$
Y \sim \operatorname{bin}(m, \pi)
$$

e

$$
g(\mu)=\beta^{\prime} X
$$

onde $\beta$ é o vetor de parâmetros a ser estimado, $X$ é uma matriz de variáveis explanatórias, $\pi$ é a probabilidade de sucesso (neste caso é a probabilidade de um ovo ser parasitado), $\mu$ é o valor esperado da variável aleatória $Y$ e $g$ é uma função de ligação adequada. A função de ligação probit é dada por

$$
g\left(\mu_{i}\right)=\Phi^{-1}\left(\pi_{i}\right)=\eta_{i}
$$

onde $\Phi^{-1}(\cdot)$ é a função de probabilidade acumulada inversa da distribuição normal padrão. A função de ligação complemento log-log é dada por

$$
g\left(\mu_{i}\right)=\log \left[-\log \left(1-\pi_{i}\right)\right]=\eta_{i}
$$

Neste trabalho será utilizada a função de ligação logit, 


$$
g\left(\mu_{i}\right)=\operatorname{logit}\left(\mu_{i}\right)=\log \left(\frac{\pi_{i}}{1-\pi_{i}}\right)=\eta_{i} .
$$

As expressões da esperança matemática e variância podem ser escritas

como

$$
E(Y)=m \pi=\mu
$$

e

$$
\operatorname{Var}(Y)=m \pi(1-\pi)=\frac{\mu}{m}(m-\mu)
$$

Nota-se que estando especificada a média da distribuição binomial, sua variância fica automaticamente especificada. Este fato limita sua aplicação em situações típicas de superdispersão, onde a variância observada é bem maior que a variância teórica admitida pelo modelo (Hinde \& Demétrio, 1997). Na prática, isto significa que este modelo binomial ajustado a dados com superdispersão tende a subestimar a variabilidade dos dados e, consequentemente, a superestimar a significância dos regressores do preditor linear $\eta=\beta^{\prime} X$. Isto, também, significa que o pesquisador estaria aumentando as chances de cometer o erro tipo I em testes de hipóteses, utilizando este modelo em uma situação de superdispersão. Isto indica a necessidade de se utilizarem outros modelos que levem em conta esta variação extra-binomial.

\subsubsection{Modelo Binomial Superdisperso}

Suponha, inicialmente, que a probabilidade de sucesso varia aleatoriamente entre as observações $y_{i}, i=1, \ldots, n$. Ou seja, $Y_{i}$ tem distribuição $\operatorname{bin}\left(m_{i}, P_{i}\right)$ e $P_{i}$ é uma variável aleatória $\operatorname{com} E\left(P_{i}\right)=\pi_{i}$ e $\operatorname{Var}\left(P_{i}\right)=\phi \pi_{i}\left(1-\pi_{i}\right)$, onde $\phi$ $(\geq 0)$ é um parâmetro de escala desconhecido. Assim, condicionalmente a $P_{i}, Y_{i}$ tem distribuição $\operatorname{bin}\left(m_{i}, P_{i}\right)$, com esperança e variância condicionais dadas por

$$
E\left(Y_{i} \mid P_{i}\right)=m_{i} P_{i}
$$

e

$$
\operatorname{Var}\left(Y_{i} \mid P_{i}\right)=m_{i} P_{i}\left(1-P_{i}\right)
$$

Como $P_{i}$ é uma variável aleatória não observável (ou latente), a esperança e a variância incondicionais de $Y_{i}$ podem ser obtidas, utilizando resultados da teoria de probabilidades condicionais. Assim, sendo $Z$ e $W$ variáveis aleatórias, tem-se que 


$$
E(Z)=E[E(Z \mid W)]
$$

$\mathrm{e}$

$$
\operatorname{Var}(Z)=E[\operatorname{Var}(Z \mid W)]+\operatorname{Var}[E(Z \mid W)] .
$$

Então, incondicionalmente, tem-se

$$
E\left(Y_{i}\right)=m_{i} \pi_{i}
$$

e

$$
\operatorname{Var}\left(Y_{i}\right)=m_{i} \pi_{i}\left(1-\pi_{i}\right)\left[1+\phi\left(m_{i}-1\right)\right]
$$

(ver Collett, 1991; Hinde \& Demétrio, 1998).

Na expressão (1), tem-se que a variância de $Y_{i}$ é igual àquela definida sob o modelo binomial quando $\phi=0$. Por outro lado, se as probabilidades de sucesso apresentam uma variação aleatória, ou seja, $\phi>0$, tem-se que $\operatorname{Var}\left(Y_{i}\right)$ vai estar multiplicada pelo fator $1+\phi\left(m_{i}-1\right)$. Desta forma, a variância dos dados será maior que aquela estipulada pelo modelo binomial, caracterizando a superdispersão.

Na modelagem de dados com este tipo de superdispersão, pode-se considerar para a estimação dos parâmetros, o método da quase-verossimilhança estendida, proposto por Nelder \& Pregibon (1987), que generaliza o escopo dos modelos lineares generalizados para uma gama maior de aplicações, aumentando a versatilidade destes modelos. Este método relaxa a suposição de que a distribuição da variável resposta $Y$ seja conhecida e completamente especificada, considerando então, a suposição mais fraca de que há uma relação conhecida entre a média e a variância das observações e onde o parâmetro de escala e a função de variância podem depender de parâmetros adicionais. Este método generaliza o método da quase-verossimilhança, proposto por Wedderburn (1974).

Estimativas de quase-verossimilhança podem ser obtidas maximizando - logaritmo da função de quase-verossimilhança,

$$
Q=-\frac{1}{2} \sum_{i=1}^{n}\left\{\frac{D\left(y_{i}, \mu_{i}\right)}{\phi}\right\}
$$

onde $D$ é a função deviance

$$
D(y, \mu)=-2 \int_{y}^{\mu} \frac{y-u}{V(u)} d u
$$


onde $\mu$ é a esperança de $Y$ e $V(\mu)$ é a função de variância em termos de $\mu$. Assim, fornecendo uma função de variância em termos da esperança da variável aleatória, é possivel obter estimativas de quase-verossimilhança para $\beta$.

Para obter-se estimativas de parâmetros de um modelo sob uma função de variância mais geral do tipo

$$
\operatorname{Var}\left(Y_{i}\right)=\phi_{i}(\gamma) V_{i}\left(\mu_{i}, \boldsymbol{\lambda}\right)
$$

maximiza-se a função de quase-verossimilhança estendida

$$
Q^{+}=-\frac{1}{2} \sum_{i=1}^{n}\left\{\frac{D\left(y_{i}, \mu_{i}\right)}{\phi_{i}}+\log \left[2 \pi \phi_{i} V_{i}\left(y_{i}\right)\right]\right\} .
$$

Williams (1982) propõe um método (que fornece estimativas equivalentes às estimativas de quase-verossimilhança estendida) para estimar $\phi$ solucionando iterativamente a equação

$$
X^{2}=E\left(X^{2}\right)
$$

onde $X^{2}$ é a estatística generalizada de Pearson (Collet, 1991). Executando uma iteração em (4) dentro de cada iteração na maximização de (2), até que se atinja a convergência, tem-se uma estimativa para $\phi$ : Sob convergência, tem-se que $X^{2} \equiv$ $n-p$, onde $n$ é o tamanho da amostra e $p$ é o número de parâmetros do modelo. Assim, quando se estima $\phi$, não se deve utilizar a deviance como medida de qualidade. de ajuste, uma vez que esta é assintoticamente próxima ao valor de $X^{2}$ e que $X^{2}$ está sendo forçada a ser próxima de $n-p$.

Após um primeiro ajuste para estimar $\phi$, considerando um modelo completo, fixa-se o valor de $\hat{\phi}$ e, consequentemente, fixam-se os pesos a priori $w_{i}$, onde $w_{i}=1 /\left[1+\phi\left(m_{i}-1\right)\right]$. Em seguida, podem ser ajustados modelos encaixados e utilizar a deviance em critérios de seleção de modelos, mas não para verificação da qualidade do ajuste do modelo, como já citado.

Considerando a expressão (1) nota-se que quando $m_{i}$ é igual para todo $i\left(\right.$ seja $\left.m_{i}=m\right)$, a função de variância pode ser escrita como

$$
\operatorname{Var}\left(Y_{i}\right)=m \pi(1-\pi) \phi
$$

onde $\phi$ é chamado fator de heterogeneidade e o modelo é chamado modelo com fator de heterogeneidade ou modelo de superdispersão constante. Este é um modelo computacionalmente mais simples onde o método de Williams se reduz a fixar 


$$
\tilde{\phi}=\frac{X^{2}}{n-p}
$$

isto é, não é mais necessário estimar o parâmetro $\phi$ através de um algoritmo iterativo.

\subsubsection{Modelo Binomial Truncado}

Se for considerado que o número de ovos parasitados iguais a zero não são por si só informativos, pode-se pensar em um modelo probabilístico que exclua valores nulos, assumindo somente os valores $Y_{i}=1,2, \ldots, m_{i}$. A função de probabilidade da distribuição binomial truncada no valor $Y=0$ é dada por (Johnson \& Kotz, 1962),

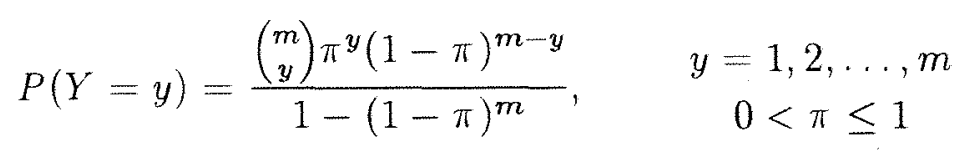

que pode ser reescrita como

$$
\begin{aligned}
f(y ; \pi) & =\exp \left\{\ln \left(\begin{array}{c}
m \\
y
\end{array}\right)+y \ln \pi+m \ln (1-\pi)-y \ln (1-\pi)-\ln \left[1-(1-\pi)^{n}\right]\right\} \\
& =\exp \left\{y \ln \left(\frac{\pi}{1-\pi}\right)-\ln \left[\frac{1-(1-\pi)^{m}}{(1-\pi)^{m}}\right]+\ln \left(\begin{array}{c}
m \\
y
\end{array}\right)\right\}
\end{aligned}
$$

Fazendo $\theta=\ln [\pi /(1-\pi)]$ tem-se $\pi=e^{\theta} /\left(1+e^{\theta}\right)$. Portanto, a função de probabilidade pode ser escrita como

$$
\begin{aligned}
f(y ; \theta, \phi) & =\exp \left\{y \theta+\ln \left(1-\frac{e^{\theta}}{1+e^{\theta}}\right)^{m}-\ln \left[1-\left(1-\frac{e^{\theta}}{1+e^{\theta}}\right)^{m}\right]+\ln \left(\begin{array}{c}
m \\
y
\end{array}\right)\right\} \\
& =\exp \left\{y \theta-\ln \left[\left(1+e^{\theta}\right)^{m}-1\right]+\ln \left(\begin{array}{c}
m \\
y
\end{array}\right)\right\}
\end{aligned}
$$

e de onde $a(\phi)=1, b(\theta)=\ln \left[\left(1+e^{\theta}\right)^{m}-1\right]$ e $c(y, \phi)=\ln \left(\begin{array}{l}m \\ y\end{array}\right)$, o que mostra que a distribuição binomial truncada é membro da família exponencial, na forma canônica (McCullagh \& Nelder, 1989). Tem-se, ainda,

$$
E(Y)=\frac{m \pi}{1-(1-\pi)^{m}}=\mu
$$


e

$$
\begin{aligned}
\operatorname{Var}(Y) & =\frac{m \pi(1-\pi)}{1-(1-\pi)^{m}}-\frac{m^{2} \pi^{2}(1-\pi)^{m}}{\left[1-(1-\pi)^{m}\right]^{2}} \\
& =\mu(1-\pi)-\mu^{2}(1-\pi)^{m} \\
& =\mu(1-\pi)\left[1-\mu(1-\pi)^{m-1}\right] \\
& =\mu\left[1-\pi-\mu+\mu-\mu(1-\pi)^{m}\right] \\
& =\mu[1-\pi-\mu+m \pi] \\
& =\mu[1-\mu+\pi(m-1)] .
\end{aligned}
$$

Pode-se ver que a expressão (7) depende do parâmetro $\pi$, e portanto não pode ser escrita apenas como função de (6), o que exige algum mecanismo para obter uma estimativa de $\pi$ que possa ser utilizada em (7) e assim implementar este modelo computacionalmente no enfoque MLG. Ridout \& Demétrio (1992) depararam com o mesmo tipo de problema na modelagem de dados de contagem através da distribuição Poisson positiva (ou Poisson truncada), onde a função de variância depende do parâmetro $\lambda$. Como a equação (6) é uma equação não-linear em termos de $\pi$, não existe uma expressão analítica direta da forma $\pi=h(\mu)$. Reescrevendo (6) na forma

$$
\pi=\frac{\mu}{m}\left[1-(1-\pi)^{m}\right]
$$

e substituindo $\pi$ do lado direito da expressão (8) por uma função $\pi^{*}=h(\mu)$, ter-se-ia uma primeira aproximação $h_{1}(\mu)$ para $\pi$. Considerando

$$
\pi^{*}=\frac{1}{m}\left(\mu-\frac{1}{\mu}\right),
$$

temos uma função cuja aproximação do verdadeiro valor de $\pi$ depende claramente do denominador da proporção, como pode ser visto na Figura 2.

Thomas e Gart (1971) fizeram um estudo da performance de alguns estimadores para o parâmetro $\pi$ da distribuição binomial truncada, sob pequenas amostras e sob denominadores pequenos. Foi demonstrado que o estimador de máxima verossimilhança de $\pi$ tende a apresentar um vício considerável, principalmente com $m \leq 8$. Uma das soluções que os autores propõem é aplicar o método de Newton-Raphson (N-R) com uma iteração, sobre os estimadores lá estudados. Ridout \& Demétrio (1992) aplicaram o algoritmo N-R à expressão de $E(Y)$ em uma iteração para obter uma solução iterativa para o parâmetro $\lambda$ da distribuição Poisson truncada, e em seguida aplicando $h_{1}(\mu)$ para obter uma aproximação refinada, 


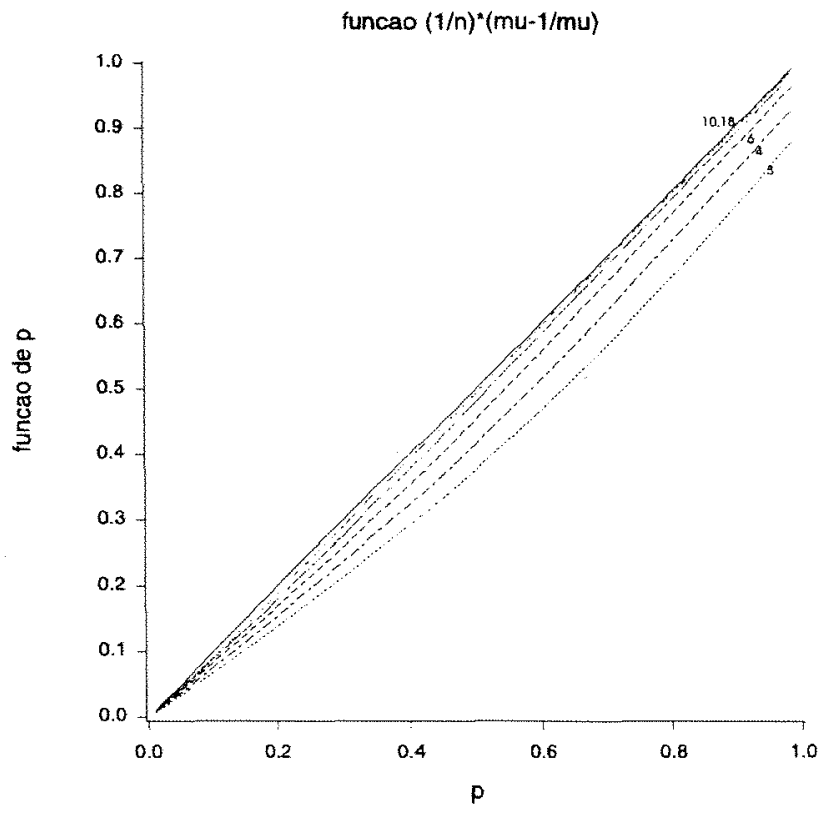

Figura 2: Gráfico da função $\pi^{*}$ para $m=3,4,6,10,18$.

$h_{2}(\mu)$. Esta aproximação é utilizada na função de variância, que é programada na forma de macros para o sistema GLIM como modelo do usuário. Esta macro passa a fazer parte do algoritmo IRLS (Iterative Reweighted Least Squares), para a estimação por máxima verossimilhança do vetor de parâmetros $\beta$. Utilizando este mesmo procedimento, aplicando (9) em (8), tem-se

$$
h_{1}(\mu)=\frac{\mu}{m}\left\{1-\left[1-\frac{1}{m}\left(\mu-\frac{1}{\mu}\right)\right]^{m}\right\} .
$$

Conforme descrito em Rustagi (1994), o algoritmo de Newton-Raphson consiste em solucionar iterativamente uma equação do tipo

$$
\pi^{(k)}=\pi^{(k-1)}-\frac{f\left(\pi^{(k-1)}\right)}{f^{\prime}\left(\pi^{(k-1)}\right)}
$$

onde $\pi^{(k)}$ e $\pi^{(k-1)}$ são estimativas de $\pi$ na $k$-ésima e (k-1)-ésima iteração, respectivamente. Considerando $f(\pi)$ como

$$
f(\pi)=\mu-\frac{m \pi}{1-(1-\pi)^{m}}
$$

obtém-se a derivada primeira de $f(\pi)$,

$$
f^{\prime}(\pi)=\frac{-m\left[1-(1-\pi)^{m}\right]+m \pi\left[m(1-\pi)^{m-1}\right]}{\left[1-(1-\pi)^{m}\right]^{2}}
$$




$$
=\frac{m\left[(1-\pi)^{m}+m \pi(1-\pi)^{m-1}-1\right]}{\left[1-(1-\pi)^{m}\right]^{2}}
$$

Utilizando (10), tem-se

$$
\begin{aligned}
\pi & =\pi_{0}-\frac{f\left(\pi_{0}\right)}{f^{\prime}\left(\pi_{0}\right)} \\
& =\pi_{0}-\frac{\left\{-\mu\left[1-\left(1-\pi_{0}\right)^{m}\right]-m \pi_{0}\right\}}{1-\left(1-\pi_{0}\right)^{m}} \times \frac{\left[1-\left(1-\pi_{0}\right)^{m}\right]^{2}}{m\left[\left(1-\pi_{0}\right)^{m}+m \pi_{0}\left(1-\pi_{0}\right)^{m-1}-1\right]} \\
& =\pi_{0}-\frac{\left[\mu-\mu\left(1-\pi_{0}\right)^{m}-m \pi_{0}\right] \cdot\left[1-\left(1-\pi_{0}\right)^{m}\right]}{m\left[\left(1-\pi_{0}\right)^{m}+m \pi_{0}\left(1-\pi_{0}\right)^{m-1}-1\right]} .
\end{aligned}
$$

Substituindo $\pi_{0}$ por $h_{1}(\mu)$ (que já é uma estimativa preliminar de $\pi$ ), tem-se

$$
\begin{aligned}
h_{2}(\mu)=h_{1}(\mu)-[\mu & \left.\left\{1-\left[1-h_{1}(\mu)\right]^{m}\right\}-m h_{1}(\mu)\right] \times \\
& \times \frac{1-\left[1-h_{1}(\mu)\right]^{m}}{m\left\{\left[1-h_{1}(\mu)\right]^{m}+m h_{1}(\mu)\left[1-h_{1}(\mu)\right]^{m-1}-1\right\}} .
\end{aligned}
$$

Ao contrário do caso da Poisson truncada, em Ridout \& Demétrio (1992), em que o erro absoluto utilizando estas aproximações era menor que 1,5× $10^{-4}$, aqui o erro absoluto é ainda considerável a um iteração. Neste caso da binomial truncada, foi fixado o número de 10 iterações para estimação preliminar de $\pi$ através da expressão (11), em uma macro programada para o sistema GLIM 4. Este valor, porém, pode ser facilmente modificado no programa. Foi também implementado na macro um controle do tamanho dos passos do algoritmo N-R, uma vez que nas primeiras iterações é comum o algoritmo divergir devido a um passo largo demais. Este parâmetro também pode ser modificado no programa. Todos os programas desenvolvidos estão apresentados no Apêndice 1.

Como a distribuição binomial truncada pertence à família exponencial de distribuições, todos os resultados assintóticos para esta família aplicam-se a este modelo (Bickel e Doksum, 1977), garantindo assim, os mesmos procedimentos inferenciais para os demais modelos lineares generalizados.

A função da deviance, após o ajuste do modelo aos dados, é dada por

$$
\begin{aligned}
d(y ; \hat{\mu}) & =2[l(y, \hat{\mu})-l(y, y)] \\
& =2 \sum_{i=1}^{n}\left\{y_{i} \ln \left(\frac{y_{i}}{\hat{\mu}_{i}}\right)+\left(m_{i}-y_{i}\right) \ln \left(\frac{m_{i}-y_{i}}{m_{i}-\hat{\mu}_{i}}\right)-\ln \left[\frac{m_{i}^{m_{i}}-\left(m_{i}-y_{i}\right)^{m_{i}}}{m_{i}^{m_{i}}-\left(m_{i}-\hat{\mu}_{i}\right)^{m_{i}}}\right]\right\},
\end{aligned}
$$


onde $y_{i}$ e $\hat{\mu}_{i}$ são os números de ovos parasitados observados e ajustados, respectivamente, e $m_{i}$ é o denominador da proporção $y_{i} / m_{i}$ da $i$-ésima observação, $i=1,2, \ldots, n$.

\subsubsection{Modelo Binomial Truncado Superdisperso}

O Modelo Binomial truncado permite modelar dados através de um modelo binomial ignorando o excesso de zeros nos dados mas, eventualmente, superdispersão devida a outras causas (probabilidade desigual de sucesso ou dados agrupados) pode estar presente. Um modelo simples que pode ser eficiente para modelar fontes de superdispersão é um modelo binomial truncado com um fator de heterogeneidade (ou um modelo binomial truncado superdisperso). A função de variância para este modelo é

$$
V(Y)=\mu[1-\mu+\pi(m-1)] \phi
$$

que é exatamente a expressão (7) multiplicada pelo parâmetro de superdispersão $\phi$ (ou fator de heterogeneidade). Uma estimativa para este parâmetro pode ser obtida após o ajuste de um modelo maximal, através da estatística qui-quadrado generalizada de Pearson médio, que é

$$
\tilde{\phi}=\frac{X^{2}}{n-p}
$$

onde $n$ é o número de observações e $p$ é o número de parâmetros estimados do modelo a serem estimados. Entretanto, a deviance média e o qui-quadrado generalizado médio não podem ser utilizados como critério para avaliar a qualidade de ajuste dos modelos, uma vez que ambos são assintoticamente equivalentes e este último está sendo forçado a ser igual à sua esperança matemática.

\subsubsection{Modelo de Mistura Bernoulli-Binomial}

Uma possível explicação para o excesso de zeros neste conjunto de dados é que algumas das fêmeas do parasita são naturalmente incapazes de parasitar os ovos do hospedeiro, possivelmente devido a sua inabilidade em se adaptar ao hospedeiro alternativo. Considere $Y$ igual ao número de ovos parasitados e $Z=I_{(A)}$, onde $I_{(A)}$ é uma função indicadora e $A$ é o evento "A fêmea parasita". Assim, condicionalmente, um modelo de mistura de distribuições seria: 


$$
\left\{\begin{array}{l}
\{Y \mid Z=1\} \sim \operatorname{Bin}(m, \pi) \\
\{Y \mid Z=0\} \equiv 0
\end{array}\right.
$$

onde $Z \sim \operatorname{Bernoulli}(\alpha)$, ou seja, $Z$ é uma variável aleatória com função de probabilidade

$$
P(Z=z)=\alpha^{z}(1-\alpha)^{1-z}, \quad z=0,1
$$

onde $\alpha$ é a probabilidade de uma fêmea parasitar. Portanto, a função de probabilidade incondicional de $Y$ pode ser escrita como

$$
P(Y=y)= \begin{cases}1-\alpha+\alpha(1-\pi)^{m}, & y=0 \\
\alpha\left(\begin{array}{c}
m \\
y
\end{array}\right) \pi^{y}(1-\pi)^{m-y}, & y=1,2, \ldots, m\end{cases}
$$

Sendo Z uma variável latente, já que não é possível saber diretamente se a fêmea não parasitou por sua incapacidade de parasitar ou não parasitou devido ao resultado aleatório modelado pela distribuição binomial, o algoritmo EM (Dempster et $a l ., 1977$ ) pode ser utilizado como um algoritmo de estimação. Basicamente, esse algoritmo consiste em um processo iterativo de 2 passos: E (Expectation), onde o valor esperado condicional de uma variável latente é calculado, e M (Maximization) é a etapa de maximização, utilizando os dados observados e os estimados do passo-E. O logaritmo da função de verossimilhança para os dados completos $(\boldsymbol{y}, \boldsymbol{z})$ é dado por

$$
\begin{aligned}
l(\pi, \alpha ; y, z) & =\ln \left[\prod_{i=1}^{n} f\left(y_{i}, z_{i}, \pi, \alpha\right)\right] \\
& =\sum_{i=1}^{n} \ln f\left(y_{i}, z_{i}, \pi, \alpha\right) \\
& =\sum_{i=1}^{n} \ln f\left(y_{i} \mid z_{i}, \pi, \alpha\right) f\left(z_{i} \mid \alpha\right) \\
& =\sum_{i=1}^{n} \ln \left[\left(\begin{array}{c}
m \\
y_{i}
\end{array}\right) \pi^{y_{i}}(1-\pi)^{m-y_{i}}\right]^{z_{i}}+\sum_{i=1}^{n} \ln \left[\alpha^{z_{i}}(1-\alpha)^{1-z_{i}}\right]
\end{aligned}
$$

Expandindo a função $\ln (\cdot)$ dentro da expressão, tem-se

$$
\begin{aligned}
l(\pi, \alpha ; y, z)= & \sum_{i=1}^{n} z_{i}\left[\ln \left(\begin{array}{l}
m \\
y_{i}
\end{array}\right)+y_{i} \ln \left(\frac{\pi}{1-\pi}\right)+m \ln (1-\pi)\right] \\
& +\sum_{i=1}^{n} z_{i} \ln \left(\frac{\alpha}{1-\alpha}\right)+n \ln (1-\alpha) \\
= & l(\pi \mid y, z)+l(\alpha \mid z) .
\end{aligned}
$$


Esta última expressão do logaritmo da função de verossimilhança mostra que a estimação de $\pi$ e $\alpha$ pode ser independentemente obtida maximizando os dois termos de $l(\pi, \alpha ; y, z)$ separadamente.

Observando a forma simples da função de verossimilhança, nota-se que $\sum_{i} z_{i}$ é uma estatística suficiente para $\alpha$ e $\sum_{i} z_{i} y_{i}$ é uma estatística suficiente para $\pi$. Uma vez que ambas são lineares em $z_{i}$, o passo-E requer apenas que a esperança condicional de $Z$ dado $Y, \pi$ e $\alpha$ seja calculada. Assim,

$$
E(Z \mid Y, \pi, \alpha)=\sum_{z=0}^{1} z P(Z=z \mid Y, \alpha, \pi)=P(Z=1 \mid Y, \alpha, \pi)
$$

Para calcular $P(Z=1 \mid Y, \alpha, \pi)$ pode-se utilizar o teorema de Bayes, obtendo-se

$$
E(Z \mid Y, \alpha, \pi)=\frac{P(Y=y \mid Z=1, \alpha, \pi) P(Z=1)}{P(Y=y \mid Z=1, \alpha, \pi) P(Z=1)+P(Y=y \mid Z=0, \alpha, \pi) P(Z=0)}
$$

que envolve probabilidades que são explicitamente definidas pelo modelo proposto. Como $P(Y \neq 0 \mid Z=0, \alpha, \pi)=0$, tem-se que

$$
E(Z \mid Y \neq 0, \alpha, \pi)=1
$$

e

$$
E(Z \mid Y=0, \alpha, \pi)=\frac{(1-\pi)^{n} \alpha}{(1-\pi)^{n} \alpha+1-\alpha}
$$

Como já foi notado, o passo-M pode ser dividido em duas partes; separando a estimação de $\pi$ e $\alpha$ :

Passo-M para $\alpha$ : Deve-se maximizar o logaritmo da função de verossimilhança para o parâmetro $\alpha$, considerando o vetor estimado $\bar{z}$ obtido no passo-E. Uma vez que a verossimilhança foi dividida em 2 termos, pode-se maximizar apenas o termo $l(\alpha \mid z)$. A solução analítica para $\tilde{\alpha}$ é

$$
\tilde{\alpha}=\frac{1}{n} \sum_{i=1}^{n} z_{i}
$$


e portanto, $\tilde{\alpha}$ é a média dos $z_{i}$ 's, obtidos no passo-E. Como pode-se ter proporçōes com diferentes denominadores, $\tilde{\alpha}$ pode ser obtida através de um programa que ajuste modelos lineares generalizados considerando como preditor linear a média geral e $z_{i}$ como variável dependente, com distribuição binomial e denominador 1 .

Passo-M para $\pi$ : O vetor $\pi$ pode ser obtido maximizando $l(\pi \mid y, z)$, o que consiste em maximizar o logaritmo da função de verossimilhança para um modelo binomial considerando $z_{i}$ como peso a priori. Se for especificado no GLIM $z_{i}$ como peso a priori, $y$ como variável resposta e um preditor linear de interesse do usuário, valores estimados $\tilde{y}$ serão obtidos e consequentemente, $\tilde{\pi}=\tilde{y} / n$.

$O$ passos $E$ e $M$ devem ser alternados até que a convergência seja obtida, considerando algum critério como

$$
\left|\frac{\alpha^{k}-\alpha^{k-1}}{\alpha^{k-1}}\right|<\epsilon
$$

onde $\alpha^{k}$ e $\alpha^{k-1}$ são estimativas de $\alpha$ nas iterações $k$ e $k-1$, respectivamente. Como valor inicial de $\alpha$ foi utilizada a proporção de zeros na amostra. Lambert (1992) sugere utilizar a estimativa $\beta^{*}$ através de um modelo Poisson truncado, como um bom valor inicial para $\beta$ em um modelo Poisson inflacionado de zeros. Como o algoritmo EM é robusto quanto a valores iniciais e, em geral, irá convergir, os valores iniciais para o vetor $\beta$ foi obtido do ajuste do modelo binomial descrito na seção 3.2.2.

\subsubsection{Modelo de Mistura Bernoulli-Binomial Superdispersa}

Este modelo é similar ao modelo da seção 3.2.6, com a diferença de que a distribuição condicional de $Y \mid Z=1$ será a versão superdispersa da distribuição binomial, conhecida como modelo com fator de heterogeneidade ou modelo de superdispersão constante (Collett, 1991). Como esta distribuição não assume uma forma explícita (e sim sua função de variância), alguma adaptação deverá ser feita no algoritmo EM. Esta modificação envolve o cálculo de $E(Z \mid Y, \alpha, \pi)$ no passo-E do algoritmo, já que não se tem a forma explícita da verossimilhança. Porém, o modelo beta-binomial (Colett, 1991) possui a mesma função de variância que o modelo descrito por Williams (1982), que por sua vez tem sua função de variância igual a do modelo com fator de heterogeneidade quando os denominadores das proporções são iguais, (Hinde \& Demétrio, 1998). Por isso, foi calculada a esperança condicional 
$E(Z \mid Y, \alpha, \pi)$ baseada na distribuição beta-binomial. A distribuição beta-binomial é obtida assumindo que uma variável aleatória $\mathrm{Y}$ tenha distribuição binomial com índice $m$ e parâmetro $\pi$, onde $\pi$ é uma variável aleatória com distribuição beta com parâmetros $\gamma$ e $\delta$. A função de probabilidade da beta-binomial é dada por

$$
P(Y=y)=\left(\begin{array}{c}
m \\
y
\end{array}\right) \frac{B(\gamma+y, m+\delta-y)}{B(\gamma, \delta)}, \quad y=0,1, \ldots, m
$$

Assim, a probabilidade de $Y=0$ pode ser escrita como

$$
P(Y=0)=\frac{B(\gamma, m+\delta)}{B(\gamma, \delta)}=\frac{\Gamma(m+\delta) \Gamma(\gamma+\delta)}{\Gamma(\delta) \Gamma(m+\gamma+\delta)}
$$

onde $B(\cdot)$ e $\Gamma(\cdot)$ são as funções Beta e Gama, respectivamente. Considerando a relação entre $\pi$, o parâmetro de superdispersão $\phi$ e os parâmetros $\gamma$ e $\delta$ (Collett, 1991), tem-se que

$$
\pi=\frac{\gamma}{\gamma+\delta} \quad \text { e } \quad \phi=\frac{1}{\gamma+\delta+1}
$$

e portanto pode-se escrever

$$
\gamma+\delta=\left(\frac{1-\phi}{\phi}\right) \quad e \quad \delta=\left(\frac{1-\phi}{\phi}\right)(1-\pi)
$$

Utilizando-se as expressões (15) e (16) pode-se obter a esperança condicional $E(Z \mid Y, \alpha, \pi)$, por substituição na expressão (12).

O modelo com fator de heterogeneidade será utilizado como parte do algoritmo EM, na estimação do vetor de parâmetros $\boldsymbol{\beta}$. Como não se pode escrever a função de verossimilhança sob este modelo de mistura, não é possível medir a qualidade do ajuste do modelo através de medidas como $-2 \ln L$ ou o critério de Akaike (1973).

\section{3 "Half-normal Plots"}

Os modelos apresentados nas sub-seções anteriores não podem ser comparados diretamente, já que alguns deles não são membros da família exponencial canônica (os modelos de mistura). Além disso, algumas medidas para comparação de modelos como -2 vezes o máximo do logaritmo da função de verossimilhança, a deviance ou o critério de informação de Akaike (AIC), são apropriados para modelos encaixados (Lindsey, 1997). 
Um maneira de verificar se o modelo está bem ajustado é utilizar o "Half-normal Plot". Este gráfico é obtido plotando-se os valores absolutos ordenados de uma medida de diagnóstico adequada contra os "half-normal scores" (as estatísticas de ordem esperadas). Um modelo adequado apresenta um padrão onde os pontos se distribuem aproximadamente em torno de uma reta. Para fornecer uma base de comparação e diminuir a subjetividade desta análise, o gráfico é aumentado com envelopes de simulação, correspondente aos "half-normal plots" de amostras simuladas atráves do modelo assumido e dos valores dos parâmetros estimados. Se o modelo é adequado é esperado que os valores observados se distribuam dentro do envelope simulado. Demétrio \& Hinde (1997) fornecem macros para o GLIM para obter "half-normal plots" para modelos lineares generalizados, incluindo alguns modelos para superdispersão.

Os passos para a construção de um "half-normal plot" são :

- ajustar um modelo e calcular $d_{(i)}$, os valores absolutos ordenados de alguma medida de diagnóstico;

- simular 19 amostras para a variável resposta utilizando o modelo ajustado e os mesmos valores das variáveis regressoras;

- ajustar o mesmo modelo para cada amostra simulada e calcular os valores absolutos ordenados do diagnóstico de interesse, $d_{j(i)}^{*}, j=1, \ldots, 19, i=1, \ldots, n$;

- para cada $i$, calcular a média, o mínimo e o máximo de $d_{j(i)}^{*}$;

- plotar estes valores e os $d_{(i)}$ observados, contra as estatísticas de ordem esperadas, dadas por

$$
\Phi^{-1}\left[\frac{\left(i+n-\frac{1}{8}\right)}{\left(2 n+\frac{1}{2}\right)}\right] \text {. }
$$

As macros de Demétrio \& Hinde (HNP) produzem "half-normal plots" para o modelo binomial e modelos binomiais superdispersos. Para os outros modelos aqui propostos é necessário fornecer macros para ajustar o modelo corrente e para simular as amostras baseadas no modelo ajustado.

Para o modelo binomial truncado foi utilizado um algoritimo de amostragem por rejeição, simulando amostras binomiais e descartando as observações iguais a zero. Este algoritmo garante que a amostra gerada tenha exatamente uma 
distribuição binomial truncada, considerando o desenvolvimento que se segue. Considere $Y$ uma variável aleatória com distribuição binomial truncada, $y$ uma observação desta v.a. e $Z$ uma variável aleatória simulada com distribuição binomial, com índice $m$ e parâmetro $\pi$. Assim, tem-se

$$
\begin{array}{rlr}
P(Y=y) & =P(Z=y \mid Z>0) & y \neq 0 \\
& =\frac{P(Z=y, Z>0)}{P(Z>0)} \\
& =\frac{P(Z=y)}{P(Z>0)} \\
& =\frac{\left(\begin{array}{l}
m \\
y
\end{array}\right) \pi^{y}(1-\pi)^{m-y}}{1-P(Z=0)} \\
& =\frac{\left(\begin{array}{l}
m \\
y
\end{array}\right) \pi^{y}(1-\pi)^{m-y}}{1-(1-\pi)^{m}} .
\end{array}
$$

O parâmetro de superdispersão estimado foi utilizado para simular as amostras binomiais truncadas superdispersas.

Para os modelos de mistura foram simuladas duas variáveis aleatórias; $Y$ com distribuição binomial (ou distribuição binomial superdispersa) e $Z$ com distribuição uniforme. Quando $Z$ era maior que $\hat{\alpha}, Y$ era tomado igual a zero, caso contrário, $Y$ era mantido inalterado. 


\section{RESULTADOS E DISCUSSÃO}

Os seis modelos estudados aqui foram aplicados aos dados do ensaio de controle biológico. O problema de como comparar modelos probabilísticos diferentes foi resolvido através do uso dos "half-normal plots", descrito na sub-seção anterior. Nas Figuras 3, 4 e 5 estão apresentados os "half-normal plots" para os modelos binomial, binomial truncado, mistura Bernoulli-binomial e suas respectivas versões superdispersas, aplicados aos dados das linhagem AA (dados que não contêm zeros). Como se poderia esperar, o modelo binomial não se ajustou bem, claramente sugerindo alguma fonte de superdispersão. O gráfico para o modelo binomial truncado apresenta o mesmo padrão. Isto já era esperado, já que quando denominador da binomial $m$ é grande, o denominador na expressão (6) fica próximo de 1 e assim o valor esperado da distribuição binomial truncada fica bastante próximo ao valor esperado da distribuição binomial. O mesmo raciocínio pode ser considerado para o modelo de mistura Bernoulli-binomial, onde a ausência de zeros reduz o modelo ao modelo binomial.

Os modelos binomial superdisperso e binomial truncado superdisperso mostram-se melhores que os modelos anteriores, onde quase todos os pontos estão dentro dos envelopes simulados. O mesmo padrão pode ser visto para o modelo Bernoulli-binomial superdisperso, que mostra os pontos residuais dentro do envelope. Este resultado também é esperado, uma vez que os dados desta linhagem não contêm zeros e portanto o modelo de mistura deve apresentar resultados similares aos obtidos pelo modelo binomial superdisperso.

Nas Figuras 6, 7 e 8 estão apresentados os "half-normal plots" para os modelos binomial, binomial truncado, mistura Bernoulli-binomial e suas respectivas versões superdispersas, aplicados aos dados das linhagem DA (dados que estão inflacionados de zeros). Já era esperado o mal ajuste do modelo binomial devido ao excesso de zeros e à superdispersão inerente aos dados. Comparando a magnitude dos resíduos padronizados de Pearson para os modelos binomial e binomial truncado, pode-se notar que uma parte da superdispersão devido ao excesso de zeros foi 

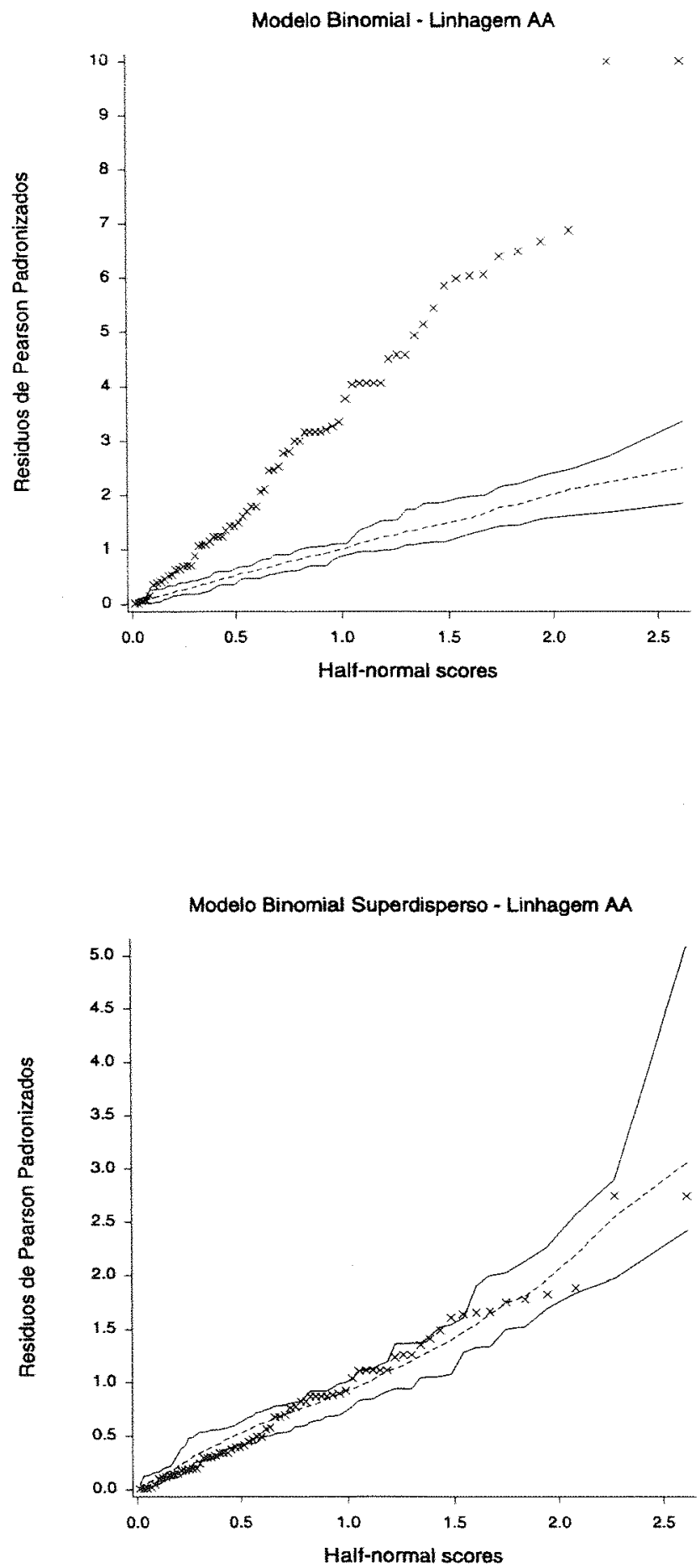

Figura 3: "Half-normal plots" para os modelos binomial e binomial superdisperso, ajustados aos dados da linhagem $\mathrm{AA}$. 


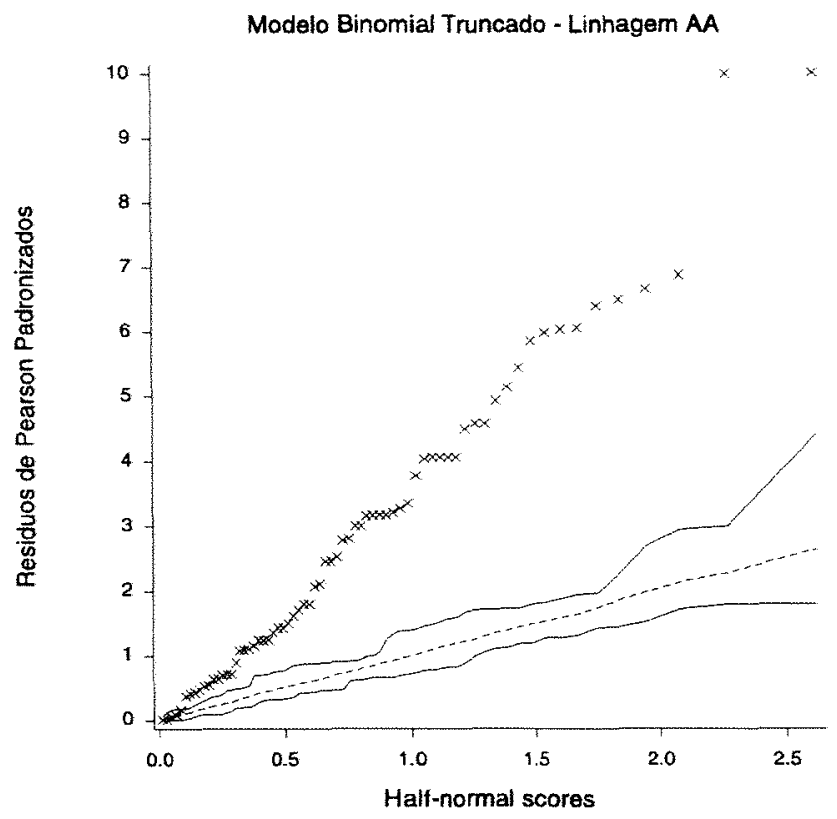

Modelo Binomial Truncado Superdisperso - Linhagem AA

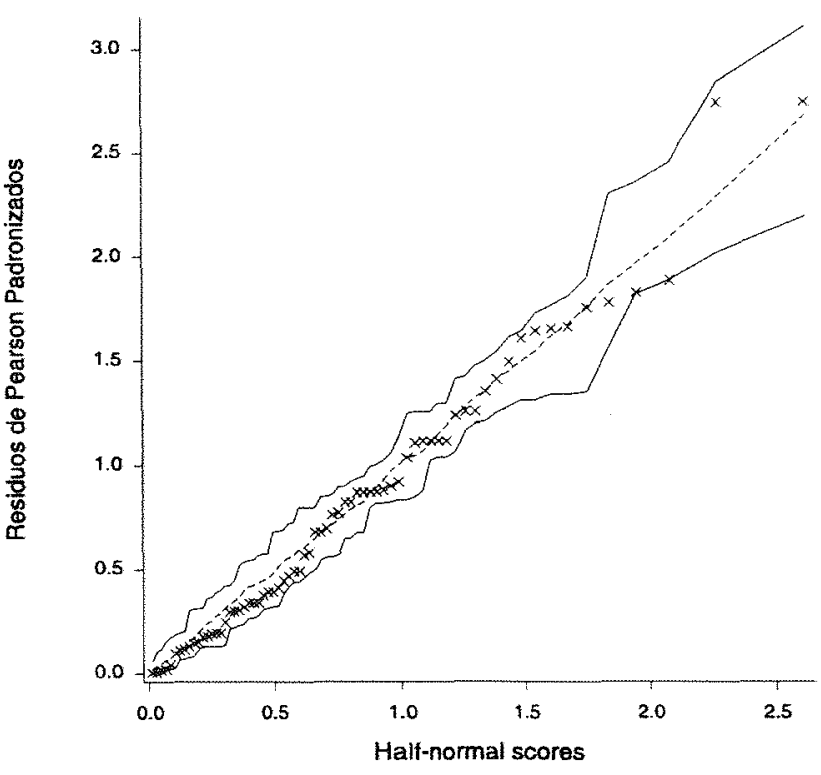

Figura 4: "Half-normal plots" para os modelos binomial truncado e binomial truncado superdisperso, ajustados aos dados da linhagem AA. 

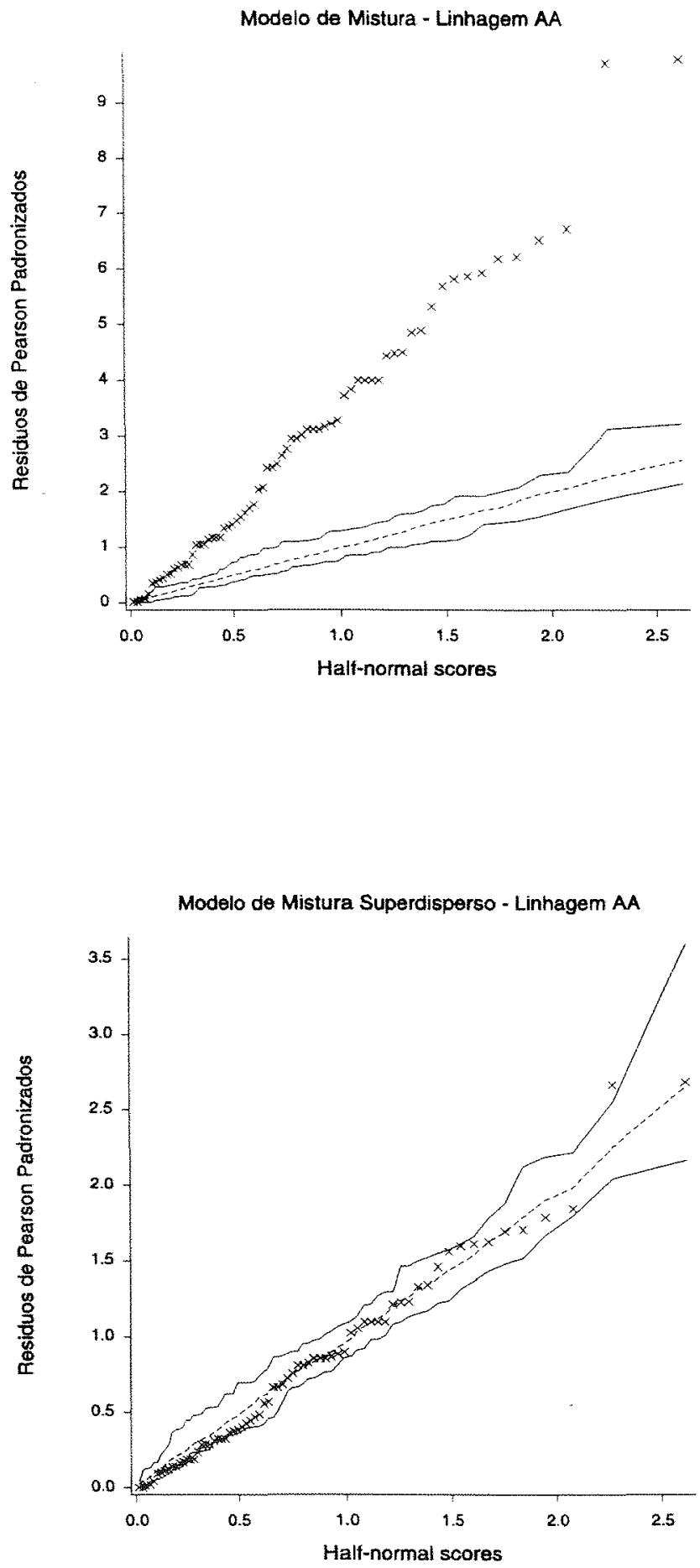

Figura 5: "Half-normal plots" para os modelos de mistura Bernoulli-binomial e Bernoulli-binomial superdisperso, ajustados aos dados da linhagem AA. 
ajustada através do modelo binomial truncado, pois os zeros foram eliminados. Entretanto, a outra parte da superdispersão, devida a outras fontes de variabilidade não pôde ser modelada, já que a função de variância para o modelo binomial truncado continua definida apenas em função do valor esperado da resposta (e pela probabilidade de sucesso $\pi$ ). Nenhum dos modelos de mistura parece ter ajustado os zeros. O modelo de mistura Bernoulli-binomial superdisperso ajustou uma grande parte dá superdispersão, mas o gráfico indica claramente a evidência de falta de ajuste.

O modelo binomial superdisperso apresentou um melhor ajuste que os modelos de mistura, mas o claro padrão no "half-normal plot" (os resíduos cruzando os limites do envelope simulado) indica problemas com este modelo, que não foi desenvolvido para modelar excesso de zeros. $O$ melhor ajuste foi obtido com o modelo binomial truncado superdisperso, onde quase a totalidade dos pontos se distribuem dentro do envelope simulado. Este modelo parece ser um boa maneira conjunta para modelagem de dados inflacionados de zeros na presença de outras fontes de superdispersão, quando o interesse reside somente sobre os dados positivos. Apesar da falha do modelo de mistura na modelagem destes dados, em alguns casos ele pode ser mais informativo que o modelo binomial truncado, uma vez que não é necessário excluir os zeros e é possível estender este modelo para modelar o processo que gera o excesso de zeros. Além disso, se fosse ajustado um modelo saturado para o parâmetro $\alpha$, este iria reproduzir o número de zeros para cada número de fêmeas estudado. Isto explica porquê o modelo binomial truncado superdisperso ajustou melhor que o modelo de mistura Bernoulli-binomial; a proporção observada de zeros não é a mesma para cada número distinto de fêmeas e ajustando um modelo de mistura básico, torna esperado o fato deste não apresentar um bom ajuste.

Escolhendo o modelo binomial truncado superdisperso como o modelo mais adequado, algum critério deve ser utilizado para comparar o ajuste de modelos com diferentes preditores lineares. Para modelos superdispersos baseados em estimativas de quase-verossimilhança, pode ser utilizada a razão da diferença de deviances dividida pela deviance média de um modelo maximal adequado (Jørgensen, 1997),

$$
F=\frac{\left(D_{2}-D_{1}\right) /\left(f_{2}-f_{1}\right)}{D_{m} / f_{m}}
$$

onde $D_{1}$ é a deviance de um modelo encaixado em um modelo maior com deviance $D_{2}, f_{1}$ e $f_{2}$ são os graus de liberdade associados com estes modelos e $D_{m}$ é a deviance do modelo maximal com $f_{m}$ graus de liberdade. A razão- $F$ é distribuida assintoticamente como $F_{\left(f_{2}-f_{1}\right) ; f_{m}}$. Fitzmaurice (1997) sugere o uso do Critério de 

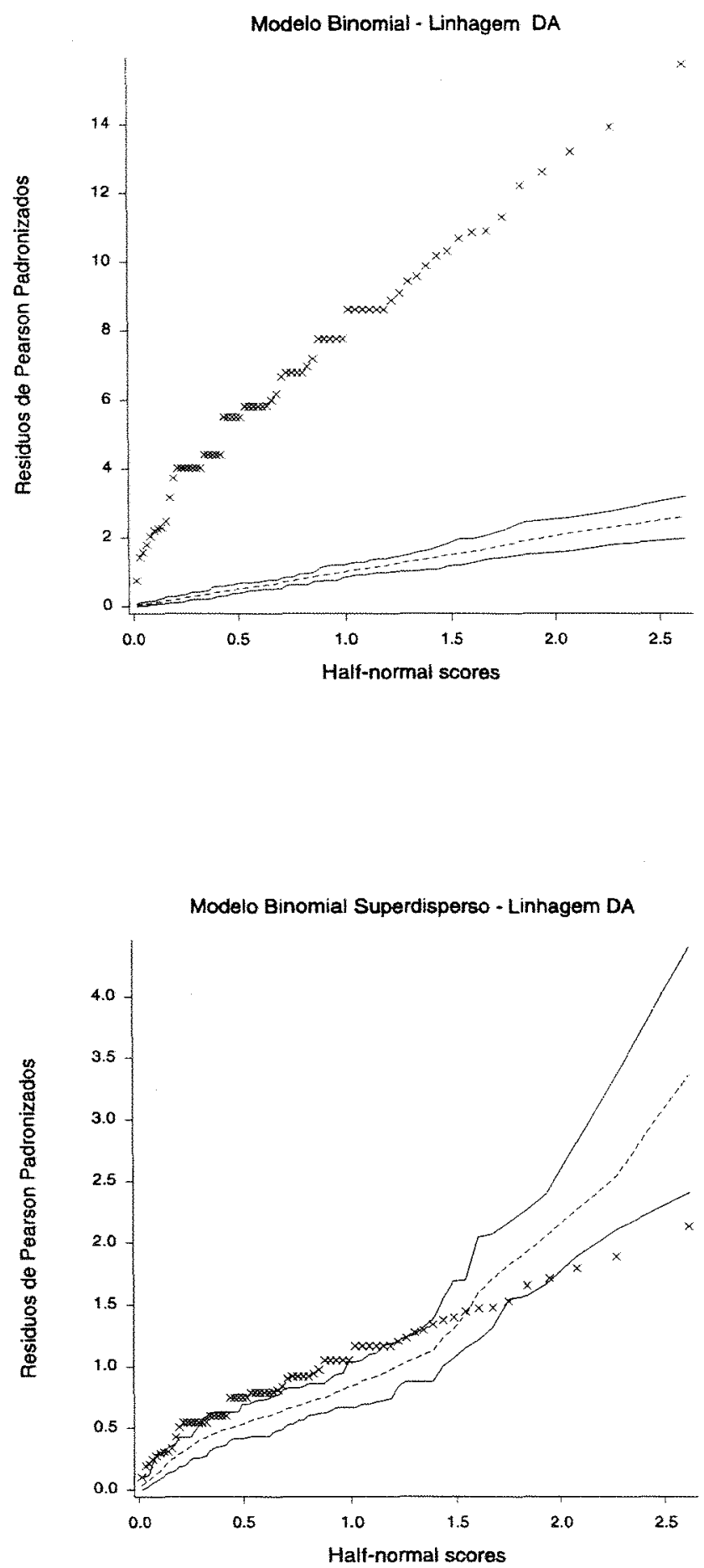

Figura 6: "Half-normal plots" para os modelos binomial e binomial superdisperso, ajustados aos dados da linhagem DA. 

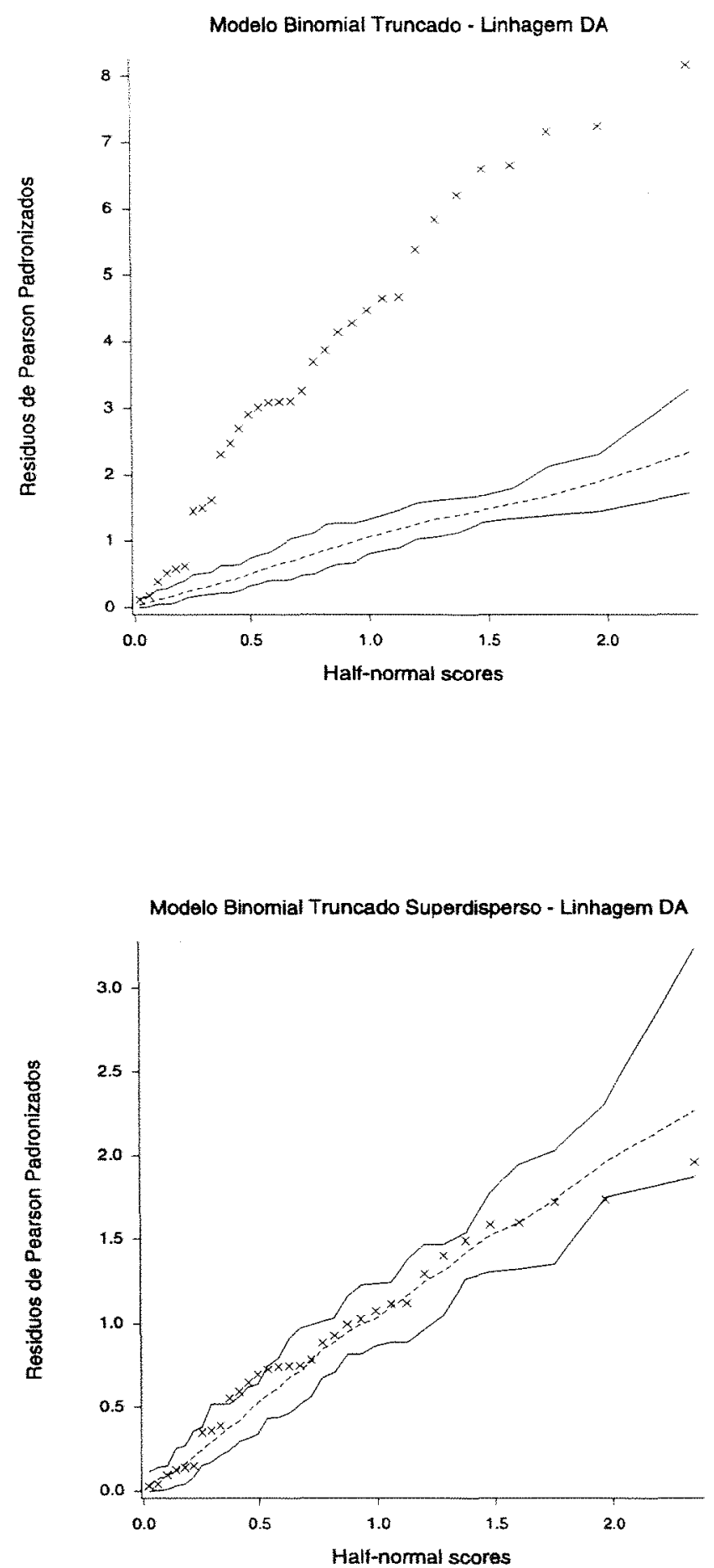

Figura 7: "Half-normal plots" para os modelos binomial truncado e binomial truncado superdisperso, ajustados aos dados da linhagem DA. 

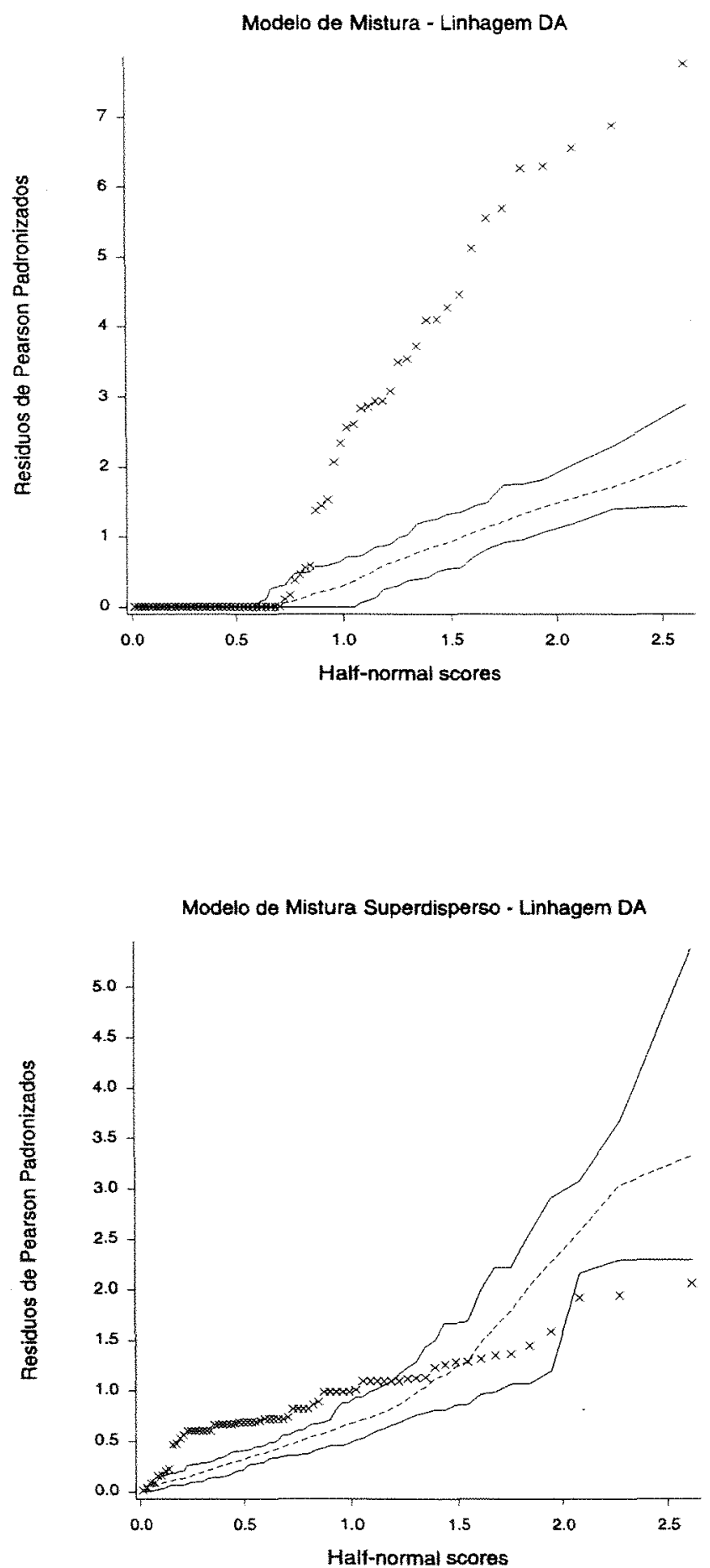

Figura 8: "Half-normal plots" para os modelos de mistura Bernoulli-binomial e Bernoulli-binomial superdisperso, a justados aos dados da linhagem DA. 


\begin{tabular}{lrrrrr}
\hline \hline \multicolumn{1}{c}{ Modelo } & AIC & Deviance & g.l. & \multicolumn{1}{c}{ F } & \multicolumn{1}{c}{ valor-P } \\
\hline Linear & -23.26 & 114.740 & 68 & - & - \\
Quadrático & -52.57 & 83.426 & 67 & 34.928 & $1.322 \times 10^{-7}$ \\
Cúbico & -60.37 & 73.632 & 66 & 8.781 & 0.0040 \\
Quártico & -59.50 & 72.501 & 65 & 1.014 & 0.3177 \\
\hline \hline
\end{tabular}

Tabela 1: Algumas estatísticas de qualidade do ajuste de alguns polinômios ortogonais, ajustados para a linhagem AA.

\begin{tabular}{lrrrrr}
\hline \hline \multicolumn{1}{c}{ Modelo } & AIC & Deviance & g.l. & \multicolumn{1}{c}{$\mathrm{F}$} & valor-P \\
\hline Linear & -16.10 & 47.897 & 31 & - & - \\
Quadrático & -14.82 & 47.178 & 30 & 0.6777 & 0.4173 \\
Cúbico & -30.28 & 29.718 & 29 & 16.4562 & 0.0003 \\
Quártico & -28.29 & 29.708 & 28 & 0.0094 & 0.9234 \\
\hline \hline
\end{tabular}

Tabela 2: Algumas estatísticas de qualidade do ajuste de alguns polinômios ortogonais, ajustados para a linhagem DA.

Informação de Akaike Modificado (Akaike, 1973), onde a deviance é penalizada pelo número de parâmetros que estão estimados no modelo e dada uma classe de modelos competitivos, o modelo selecionado deve minimizar

$$
\mathrm{AIC}=-2 \log (\text { verossimilhaça maximizada })+2 \text { (número de parâmetros }) .
$$

Vários modelos polinomiais foram ajustados para ambas as linhagens e os resultados estão apresentados na Tabela 1 (linhagem AA) e Tabela 2 (linhagem DA), com os valores para as deviances, índice AIC, estatísticas $F$ e suas respectivas probabilidades de significância. Para ambas as linhagens o modelo cúbico apresentou o melhor ajuste, com valores-P menores que 0,01 .

Estes modelos cúbicos foram ajustados utilizando polinômios ortogonais no GLIM4, já que esta é uma maneira numericamente mais estável para estimação de parâmetros em modelos polinomiais. A dificuldade em utilizá-los é que os coeficientes ajustados não fornecem a forma algébrica do polinômio (Francis, Green \& Payne, 1993). Para obter um intervalo de confiança para o número máximo de ovos parasitados, utiliza-se o comando PREDICT do programa GLIM4. Isto permite o cálculo dos valores esperados de predição do preditor linear e suas respectivas variâncias. Com isso é possivel calcular um intervalo de confiança para o valores estimados do preditor linear utilizando, 


$$
\left[\hat{\eta} \pm t_{d . f ; \alpha \alpha} \widehat{V(\hat{\eta})}\right]
$$

onde $\hat{\eta}$ é o valor predito do preditor linear e $\widehat{V(\hat{\eta})}$ é sua variância. Aplicando o inverso da função logit $\left(m \exp (\cdot)[1+\exp (\cdot)]^{-1}\right)$ para os limites deste intervalo de confiança, obtém-se um intervalo de $100 \cdot(1-\alpha) \%$ de confiança para o número máximo de ovos parasitados.

Para a linhagem AA o intervalo com $95 \%$ de confiança é $(116,51$; $122,94)$ para o número máximo de ovos parasitados $\hat{\mu}=120$, utilizando 42 fêmeas. O mesmo intervalo para a linhagem DA é $(80,87 ; 103,48)$, para $\hat{\mu}=93,13$ utilizando 52 fêmeas. Portanto, pode ser visto que a mudança do hospedeiro natural (Diatraea saccharalis) para o hospedeiro alternativo (Anagasta kuehniella) leva a uma redução de aproximadamente 22 por cento no número máximo de ovos parasitados.

Os "half-normal plots" parecem ser uma ferramenta bastante útil para a escolha do modelo probabilístico que deve ser utilizado para a modelagem de dados, especialmente quando utilizados conjuntamente com envelopes de simulação. Mas a escolha do modelo também deve estar baseada na importância ou no interesse sobre os zeros dos dados. Para os conjuntos de dados estudados aqui, as proporções zeros não são informativas, já que o interesse é sobre o número máximo de ovos parasitados dado que pelo menos um ovo tenha sido parasitado. Quando há o interesse explícito na proporção de zeros os modelos de mistura têm a vantagem de poder incorporar covariáveis que possam influenciar a probabilidade de ocorrência de zeros.

A aplicação destes modelos não se restringem à análise de dados de ensaios biológicos. Algumas possíveis áreas de aplicação são:

- uma característica de qualidade é medida como uma proporção ou como uma contagem binomial (número de componentes defeituosos em um lote, por exemplo). Se esta característica é resultado de um processo com uma percentagem de defeituosos muito baixa (bastante típico em processos industriais no estado 'sob controle'), haverá muitos zeros registrados. Entretanto, pode ser que alguns fatores possam afetar o processo, resultando em alguns valores positivos no processo de contagem (estado 'fora de controle');

- análise de regressão logística em estudos epidemiológicos retrospectivos, com baixa ocorrência de uma característica biológica em particular, na população;

- modelagem da proporção de germinação de sementes em várias condições, por exemplo: solo ácido ou árido. 


\section{CONCLUSÕES}

Nesta dissertação foram propostos 4 novos modelos para modelagem de dados de proporções com superdispersão e juntamente com 2 modelos descritos na literatura, foram aplicados a um conjunto de dados de um ensaio de controle biológico. Para os modelos aqui propostos, foram programadas as macros para serem utilizadas no programa GLIM4. Como critério de comparação da adequacidade dos modelos, foram utilizados "half-normal plots" com envelopes simulados, assim como outras medidas de qualidade de ajuste de modelos.

O modelo binomial truncado superdisperso apresentou o melhor ajuste aos dados. Como preditor linear para este modelo, foram utilizados polinômios ortogonais de terceira ordem que permitiu estimar o número máximo de ovos parasitados para cada linhagem, intervalar e pontualmente. Com estes resultados o entomologista poderá prever recursos do laboratório para este tipo de ensaio, otimizando assim a utilização do recurso financeiro e do tempo para execução do ensaio.

Apesar de os modelos de mistura não terem sido adequados aos dados, em outras applicaçōes ou tipos de ensaios, estes podem ser úteis, dando uma maior informação ou conhecimento ao pesquisador sobre o processo gerador de zeros na amostra. 


\section{REFERÊNCIAS BIBLIOGRÁFICAS}

Agresti, A. Categorical data analysis. New York: John Wiley and Sons, 1990. $558 \mathrm{p}$.

Aitkin, M.A., Anderson, D.A., Francis, B., Hinde, J.P. Statistical modelling in GLIM. Oxford: Oxford University Press, 1989.

AKAIKE, H. Information theory and an extension of the maximum likelihood principle. In Proc. 2nd Int. Symp. Information Theory (eds B.N. Petrov and E. Csàki). Budapest: Akademiai Kiàdo, 1973.

Bickel, P.J., Doksum, K.A. Mathematical Statistics. Oakland: Holden-day, Inc., 1977. 492p.

Botelho, P.S.M. Tabela de vida ecológica e simulação da fase larval da $D i$ atraea saccharalis (Fabr., 1794) (Lep.: Pyralidae). Piracicaba, 1985. Tese (Doutorado) - ESALQ/USP.

Brooks, S.P., Morgan, B.J.T., Ridout, M.S., PACK, S.E. Finite mixture models for proportions. Biometrics, 53, p.1097-1115, 1997.

Collett, D. Modelling binary data. London: Chapman-Hall, 1991.

Cordeiro, G.M. Modelos Lineares Generalizados. Campinas: VII SINAPE, 1986. $286 \mathrm{pp}$.

Demétrio, C.G.B. Modelos Lineares Generalizados na Experimentação Agronômica, 5ํ SEAGRO e 38 a RRBRAS. Porto Alegre: DE/UFRGS, 1993. $125 \mathrm{p}$. 
Demétrio, C.G.B., Hinde, J. Half-Normal plots and overdispersion. GLIM Newsletter, 27, p.19-26, 1997.

Dempster, A.P., Laird, N.M., Rubin, D.B. Maximum likelihood from incomplete data via the EM algorithm (with discussion). Journal of the Royal Statistical Society, series B, 39, p.1-38, 1977.

Dobson, A.J. An introduction to generalized linear models. London: Chapman-Hall, 1990. 174p.

Finney, D.J. Probit Analysis. Cambridge: Cambridge univ. press, 1947.

Fioravante, A.M. Alguns modelos para dados de contagem resultantes de experimentos com cultura de tecidos. Piracicaba, 1995. 136p. Dissertação (Mestrado) - ESALQ/USP.

Fitzmaurice, G. Model selection with overdispersed data. The Statistician, 46, p.81-91, 1997.

Francis, B., Green, M., Payne, C. The Glim system: generalized linear iteractive modelling. Oxford: Oxford University Press, 1993. 821p.

Grizzle, J.E., Starmer, C.F., Koch, G.G. Analysis of categorical data by linear models. Biometrics, 25, p. 489-504, 1969.

Healy, M.J.R. GLIM : An introduction. Oxford: Oxford University Press, 1988. 130p.

HindE, J.P. Macros for fitting overdispersion models. GLIM Newsletter, 26, p. 10-9, 1996.

Hinde, J.P., DemÉtrio, C.G.B. Modelling with overdispersion. In: International Workshop on Statistical Modelling, 11., Orvieto, 1996. Proceedings. Statistical Modelling. Orvieto, 1996. p.200-7.

Hinde, J.P., Demétrio, C.G.B. Overdispersion: models and estimation. Computational Statistics and Data Analysis, 27, p.151-170, 1997.

Hosmer, D.W., Lemeshow, S. Applied Logistic Regression. New York: John Wiley \& Sons, 1989. 307p. 
Johnson, N.L., Kotz, S. Distributions in Statistics: Discrete Distributions. New York: Houghton Miffin, 1962.

Jørgensen, B. The Theory of Dispersion Models. London: Chapman \& Hall, 1997. 225p.

LAmbert, D. Zero-Inflated Poisson regression, with an application to defects in manufacturing. Technometrics, 34, p.1-14, 1992.

McCullagh, P., Nelder, J.A. Generalized linear models. 2ed. London: Chapman-Hall, 1989.

Morgan, B.J.T. Analysis of Quantal Response Data. London: ChapmanHall, 1992.

Nelder, J.A., Pregibon, D. An extended quasi-likelihood function. Biometrika, 74, p.221-232, 1987.

Nelder, J.A., Wedderburn, R.W.M. Generalised linear models. Journal of Royal Statistical Society Serie A, 135, p. 370-84, 1972.

PARra, J.R.P. Técnicas de criação de Anagasta kuehniella, hospedeiro alternativo para a produção de Trichogramma. In: Parra, J.R.P., Zucchi, R.A. (Eds.). Trichogramma e o controle biológico aplicado. Piracicaba, FEALQ, 1997. Cap. 4, 121-50.

PARra, J.R.P., ZuCChI, R.A. (Eds.). Trichogramma e o controle biológico aplicado. Piracicaba, FEALQ, 1997. 324p.

Ridout, M.S., Demétrio, C.G.B. Generalized linear models for positive count data. Revista de Matemática e Estatística, 10, p.139-148, 1992.

Rustagi, J.S. Optimization Techniques in Statistics. San Diego: Academic Press, 1994.

Thomas, D.G., Gart, J.J. Small sample performance of some estimators of the truncated binomial distribution. Journal of the American Statistical Association, 66, p.169-177, 1971.

WedDERBURN, R.W.M. Quasi-likelihood functions, generalized linear models and the Gauss-Newton method. Biometrika, 61, p.439-47, 1974. 
Williams, D.A. Extra-binomial variation in logistic linear models. Applied Statistics, 31, p.144-148, 1982.

ZoCCHI, S.S. Misturas de modelos "Logit", "Probit" e "Complemento log-log”. Piracicaba, 1993. 129p. Dissertação (Mestrado) - ESALQ/USP. 


\section{APENNDICE 1}

\section{Programas e Macros}


$\$ c$

PROGRAMA : ANLSE_2.GLM

DESCRTCAO: Este programa faz a analise dos dados de FASE2.GLM.

Sexao ajustados modelos assumindo a distribuicao binomial.

AUTOR: Afranio Vieira - WSOR/Un.EXETER/UK

DATA: $10 / 10 / 97$

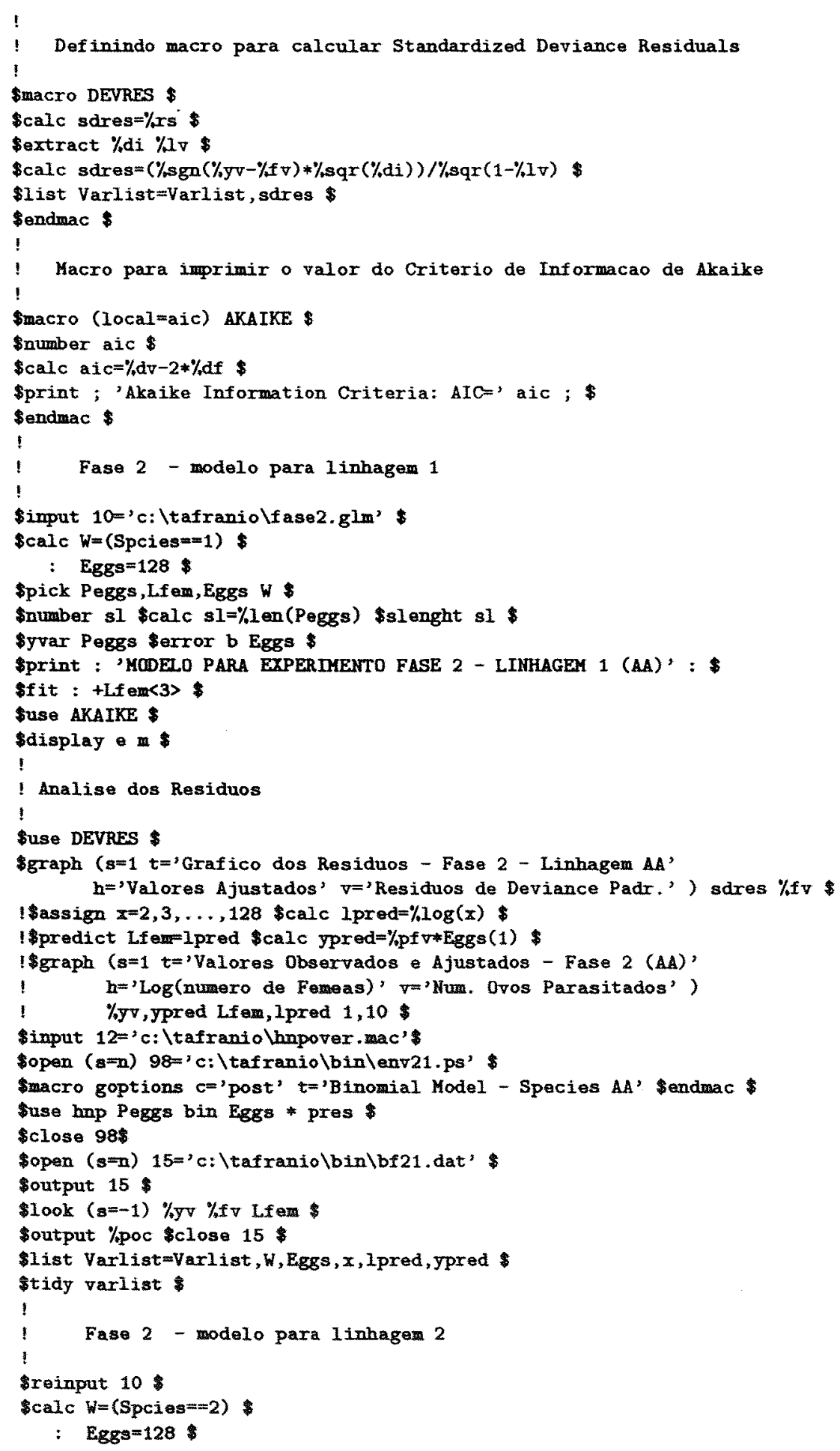


\$pick Peggs,Lfem,Eggs Peggs, Lfem,Eggs $W$

\$number sl \$calc sI=\%len(Peggs) \$slenght sl \$

\$yvar Peggs \$error b Eggs \$

\$print : 'MODEL Para EXPERTMENTO FaSE 2 - LINHAGEM 2 (DA)' : \$

\$fit : +Lfem<3>\$

\$use AKAIKE \$

Sdisplay a

\$use DEVRES

\$graph ( $s=1 \quad t={ }^{\prime}$ 'Grafico dos Residuos - Fase 2 - Linhagem DA'

h='Valores Ajustados' $v=$ 'Residuos de Deviance Padr.' ) sdres \% fv

! \$assign $x=2,3, \ldots, 128$ \$calc lpred $=\% \log (x)$ \$

Ispredicted Lfem=lpred \$calc ypred=\%pf $\nabla *$ Eggs (1) \$

I graph ( $s=1$ t='Valores Observados e Ajustados - Fase 2 (DA)"

! h='Log(numero de Femeas)' v='Num. Ovos Parasitados')

! \%yv,ypred Lfem, lpred 1,10\$

\$reinput $12 \$$

\$open (s=n) 98='c: \tafraniolbin\env22.ps' \$

\$macro goptions $c=$ 'post' $t=$ 'Binomial Model - Species DA' Sendmac

\$use hnp Peggs bin Eggs * pres \$

\$close 98 s

\$open $(s-n) 15=' c: \backslash$ tafranio $\backslash$ bin $\backslash$ bf22.dat'

Soutput 15

\$look $(s=-1) \% y v$ f f Lfem $\$$

\$output \%poc \$close $15 \$$

Slist $\operatorname{varlist=varlist,~} W$, Eggs, Ipred, ypred, $x$

\$tidy varlist

\$delete varlist \$

\$return \$

$\$ \mathrm{c}$

PROGRAYA: ANLSE_3.GLM

DESCRICAO: Este programa faz a analise dos dados de FASE2.GLM.

Serao ajustados modelos assumindo a distribuicao binomial

truncada. 0 modelo binomial truncado pertence 'a familia

exponencial de distribuicoes e sera fornecida ao GLIY como

um modelo do usuario, atraves da macro TBIN.MAC.

Como trata-se de um modelo binomial truncado para valores de PEGGS $=0$, AS OBSERVACOES SOB ESTA CONDICAO MAO SERAO CONSIDERADAS NO MODELO.

AUTOR: Afranio Vieira - WSOR/Un. EXETER/UK

DATA : $10 / 11 / 97$

\$warn off

I

! Definindo macro para calcular standardized Deviance Residuals

\$racro DEVRES

bcalc sdresm\%s

Sextract \%di $\% 17$

\$calc sdres $=(\% \mathrm{sgn}(\% \mathrm{gv}-1 / \mathrm{fv}) * \% / \mathrm{sq} x(\% \mathrm{di})) / \%$ sqx $(1-\% 1 \mathrm{v})$

list Varlist=Varlist, sdres

Sendinac

!

! Hacro para imprimir o valor do Criterio de Informacao de Akaike

ISwacro (locaI=aic) AKAIKE

ITramber aic

I calc aic $=\%$ d $\nabla-2 \% \%$ df

IPrint ; 'Akaike Inforation Criteria: AIC=' aic ;

15endmac.

Fase 2 - odelo para Iinhagem 1 
\$input 10='c: \tafranio \fase2.glm' \$

$\$$ calc $W=($ SPCIES $=1) *($ PEGGS $>0)$

: EGGS $=128$

\$pick Peggs,Lfem,Eggs Peggs,Lfem,Eggs W

\$number sl \$calc sl=\%len(Peggs) \$slenght sl \$

\$yvar PEGGS \$

\$input $11=$ 'c: \tafranio ttbin.mac's

Snumber $A=1$

Suse SETDEN EGGS A

\$print : 'MODELO PARA EXPERIMENTO FASE 2 - LIMHAGEM 1 (AA)' : \$

$\$$ Iit LFEM<3>

I\$use AKAIKE

\$use DEVRES

\$graph ( $s=1 \mathrm{t}=$ 'Grafico dos Residuos - Fase 2 (AA)' $h=$ 'Valores Ajustados' v='Residuo de Deviance Padr.") sdres \%fv

I\$assign $x=2,3, \ldots, 128 \$$ calc xpred=\%/log $(x) \$$

I\$predict Lfemexpred \$calc ypred=\%pf $\nabla *$ Eggs (1)

! \$graph ( $s=1$ t='Valores observados e Ajustados - Fase 2 (AA)'

! $\quad h=$ 'Log (numero de femeas)' $v=$ 'numero de ovos parasitados')

! \%yv, \%,fv Lfem,Lfem 1,10

\$display m e

\$input 20='c: \tafraniolhnpover.mac' \$

input 21='c: \tafraniolhnptbin.mac'

Smacro goptions $c=$ 'post' $t=$ 'Truncated Binomial Model - Species AA'

\$endmacro

Sopen ( $s=n)$ 98m' $c$ : \tafranio \tbin\tbin1.ps'\$

\$use hnp peggs tbin eggs * pres

$\$ c l o s e 98$

Sopen $(s=n) 15=$ 'c: \tafranio \tbin \tbf21.dat'

Soutput 15

$\$ 100 k(s=-1) \%$ yv \% fv Lfem

\$output \%poc \$close 15 \$

Sprint ::

$\$ 1$ ist Varlist=Varlist, W, Eggs, $x$, xpred, ypred

tidy varlist

1

!

Fase 2 - modelo para linhagem 2

$\$ r e i n p u t 10 \$$

\$calc $W=($ SPCIES $==2) *($ PEGGS $>0)$

: Eggs=128 \$scale 0 chisq

\$pick Peggs, Lfem, Eggs Peggs, Lf em, Eggs W

Snumber sl \$calc sl=\%1en(Peggs) \$slenght sl \$

\$yvar Peggs

$\$$ reinput 11 \$

Suse SETDEN Eggs a

print : 'MODELO PARA EXPERTMENTO FASE 2 - LINHAGEM 2 (DA) " :

fit LFEM〈3>

I\$use AKaIKE \$

\$use DEVRES

\$graph ( $s=1$ t='Grafico dos Residuos - Fase 2 (DA)' h='Valores Ajustados' v-Residuo de Deviance Padr.') sdres \% $\mathrm{f}$.

! \$assign $x=2,3, \ldots, 128$ calc $\operatorname{xpred}=\% \log (x)$

I \$predicted Lfem=xpred Scalc ypred=\%pf $\nabla *$ Eggs(1)

I' Graph ( $\mathrm{s}=1$ t='Valores observados ajustados - Fase 2 (DA)'

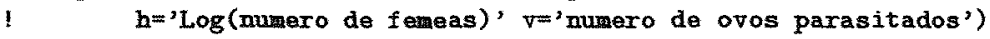

! \%yv, \%, Lfem,Lfem 1,10

odisplay

\$reinput 20 \$

\$reinput 21

Smacro goptions c='post' t='Truncated Binomial Model - Species DA'

Sendmacro

\$open $(s=n)$ 98"'c: \tafranio \tbin \tbin2.ps'

\$use hnp peggs tbin eggs * pres

close 98

\$open (s-n) 15='c: \tafranio 
Soutput $15 \$$

$\$ 100 k(s=-1) \%$ yv \%fv Lfem $\$$

Soutput \%poc \$close 15

\$ist varlist=varIist, W,EGGS, $x$, xpred, ypred \$

\$tidy varlist \$

Sdelete varlist $\$$

\$return \$

$\$ c$

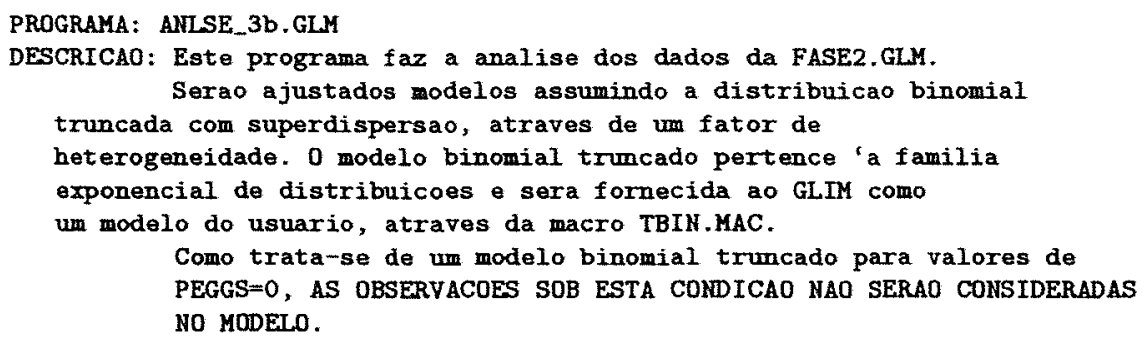

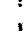

! Fase 2 - modelo para 1inhagem 1

Simput 10" 'C: (tafraniolfase2.gla'

Scalc $W=($ SPCIES $=1) *($ PEGGS $>0)$

: EGGS $=128$ क

\$pick Peggs, Lfem,Eggs Peggs,Lfem, Eggs

\$number sI calc sl=\%,len (Peggs) \$slenght sI

\$yvar PEGGS

\$input 11='c: \tafranioltbin.mac'

\$numer $A=0$ IParamentro de escala sera' estimado!

\$use SETDEN EGGS A

Sprint : 'MODELO PARA EXPERTMEatro FASE 2 - LINHAGEM 1 (AA)' :

\$fit LFEM $\langle 4\rangle$ Scalc $\% \mathrm{~s}=\%$ sc scale $\% \mathrm{~s}$ \$ Hodelo Maximal

\$fit LFEM<1> Suse AKAIKE

\$fit LFEM <2> \$use AKAIKE

It it LFEM<3> Luse AKAIKE

\$fit LFEM<4> Suse AKAIKE

print : 'The cubic model':

scale O c Afit LFEar<3>

\$display a 
\$assign $x=2,4, \ldots, 140$ par 70 ovos $\$ \mathrm{calc}$ ovos=128: $\operatorname{xpred}=\% \log (x) \$$

$\$$ predict 1 fem=xpred eggs=ovos \$calc ypred=\%pfv \$

\$use DEVRES \$

$\$ g r a p h$ ( $s=1 \quad t=$ 'Grafico dos Residuos - Fase 2 (AA)' $h=$ 'Valores Ajustados' v'Residuo de Deviance Padr.') sdres \% fv

! \$open ( $s=n$ ) 98='c: \tafraniolotbin\obsfit1.ps' \$

\$graph ( $s=1 \mathrm{c}={ }^{\prime}$ post' $t={ }^{\prime}$ Valores observados e Ajustados - Fase 2 (AA)' $h=$ 'Log (numero de femeas)' $v=$ 'numero de ovos parasitados')

$\% y v$,ypred L.fem, xpred 1,10

I\$close 98 \$

Pprint 'Valores preditos pelo modelo :' : \$

\$look ( $s=1$ ) xpred ypred \$

\$print : 'Preditor linear do Maximo: " \%plp(21) \$

\$print : 'Variancia do preditor linear do Maximo: " \%pl(21) : \$

\$input 20='c: \tafraniolhnpover.mac'

\$input 21='c: \tafranio hnnptbin.mac'

\$macro goptions $c=$ 'post' $t=$ 'Overdispersed Trumc. Binomial Model - Species AA'

Sendmacro \$

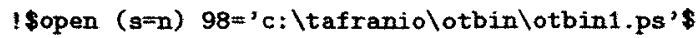

\$use hnp peggs tbin eggs \$

1\$close $98 \$$

! \$open ( $s=n) 15={ }^{\prime} c$ : \tafranio $\backslash o t b i n \backslash o t b f 21$.dat'

! \$output $15 \$$

! \$look ( $s=-1$ ) \%yv \%fv Lfem $\$$

I\$output \%poc \$close 15 \$

print ::

\$1ist Varlist=Varlist,W,Eggs, $x$, xpred, ypred

\$tidy varlist \$

Fase 2 - modelo para 1inhagen 2

Sreinput 10

$\$$ calc $W=($ SPCIES $==2) *($ PEGGS $>0)$

: $\mathrm{Eggs}=128$

\$pick Peggs, Lfem,Eggs Peggs, Lfem,Eggs W

\$number sl \$calc sl=\% Ien (Peggs) \$slenght sI

\$yvar Peggs

Sreinput 11

\$use SETDEN Eggs a

Sprint : 'MODELO PARA EXPERTMERTO FASE 2 - LIMHAGEM 2 (DA)' : \$

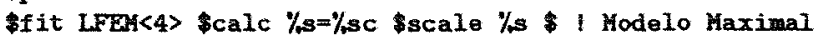

\$fit LFEM<1> \$use AKAIKE

\$fit LFEM<2> suse AKAIKE \$

ffit LFEMY3> Suse AKAIKE \$

Sf it LFEA <4> Suse AKAIKE

pprint : 'The cubic model, :

$\$ s c a l e 0$ c \$fit $1+$ LFET< $3>\$$

sdisplay $\theta$

assign $x=2,5.8484, \ldots, 125$ del oros $\$$ var 33 oros

scalc oros $=128$ : $x p r e d=\%, \log (x)$

\$predict if empred eggs=oros \$calc ypred=\%pfv

Suse DEVRES

\$graph ( $g=1$ t='Grafico dos Residuos - Fase 2 (DA)' $h=$ 'Valores Ajustados' v='Residuo de Deviance Padr.') sdres \% If

I \$open $(g=n) 98=' c$ : Itafraniolotbinlobsfit2.ps'

\$graph ( $g=1$ c='post' $t=$ 'Valores observados e Ajustados - Fase 2 (DA)' $h=$ 'Log (numero de femeas)' $\nabla=$ 'numero de ovos parasitados')

$\% y v$, ypred Lfem, xpred 1,10

$1 \$$ close $98 \$$

Sprint 'Valores preditos pelo modelo :" :

\$look ( $\mathrm{s}=1$ ) xpred ypred $\$$

Sprint : 'Preditor linear do Maximo: "\%plp(14)

\$print : 'Variancia do preditor linear do Maximo: ' \%pli(14) :

Srejuput 20

\$reiaput 21

\$racro goptions $c=$ 'post' $t=$ 'Overdispersed Trunc. Binomial Model - Species DA' 


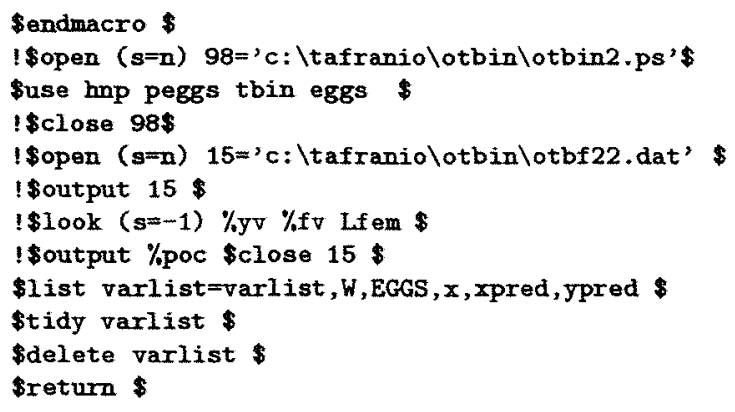

$\$ c$

PROGRAMA: ANLSE_4.GLY

DESCRICAO: Este programa faz a analise dos dados de FASE2.GLM.

Serao ajustados modelos assumindo uma mistura de distribuicoes

(Bernoulli-Binomial), atraves do algoritmo EM (em conjunto com

o algoritmo IRLS do GLIM). Este modelo e' apropriado para

dados de contagem binomial, possivelmente inflacionados de zeros.

AUTOR: Afranio Vieira - MSOR/Un. EXETER/UK

DATA : $1 / 12 / 97$

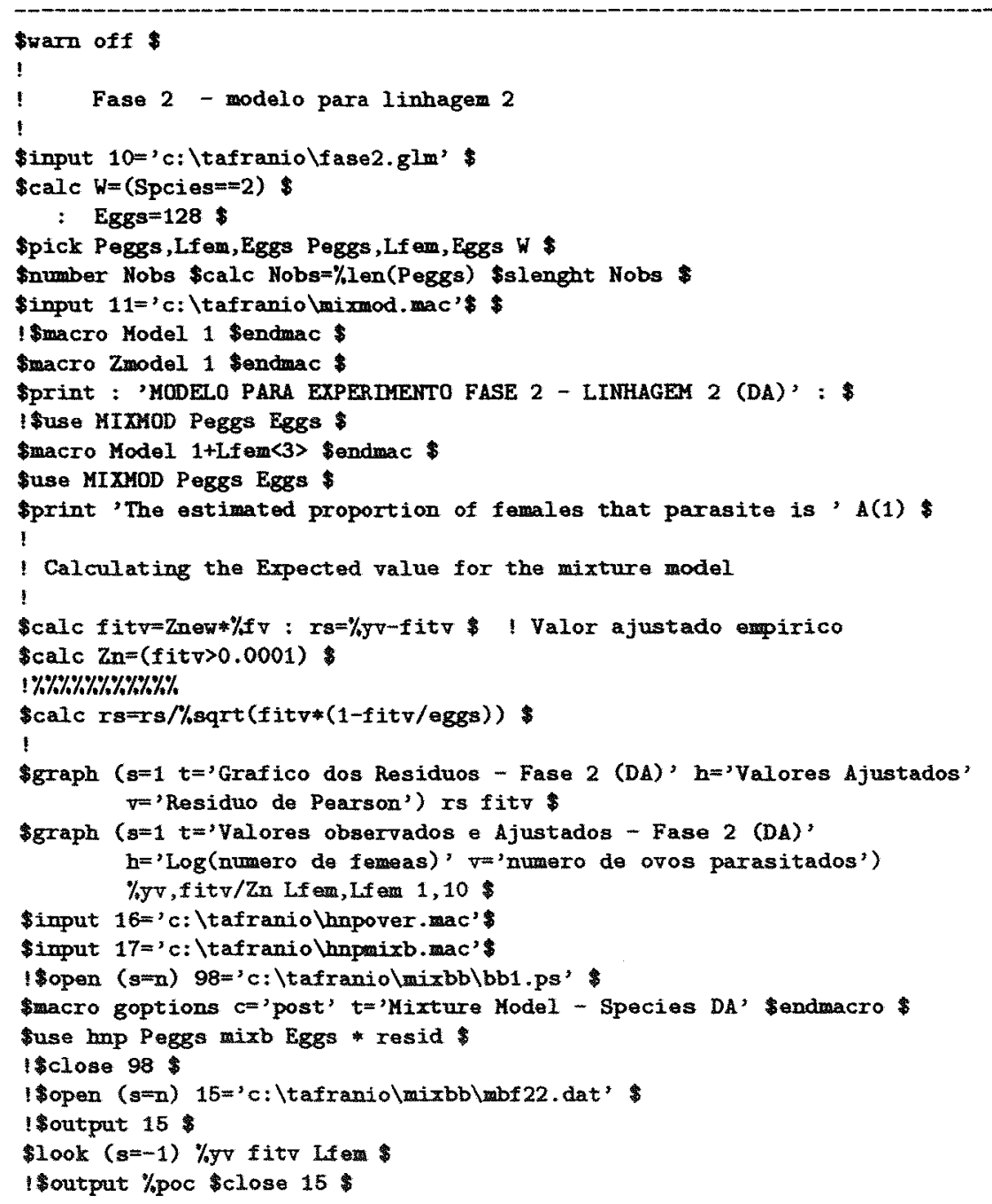




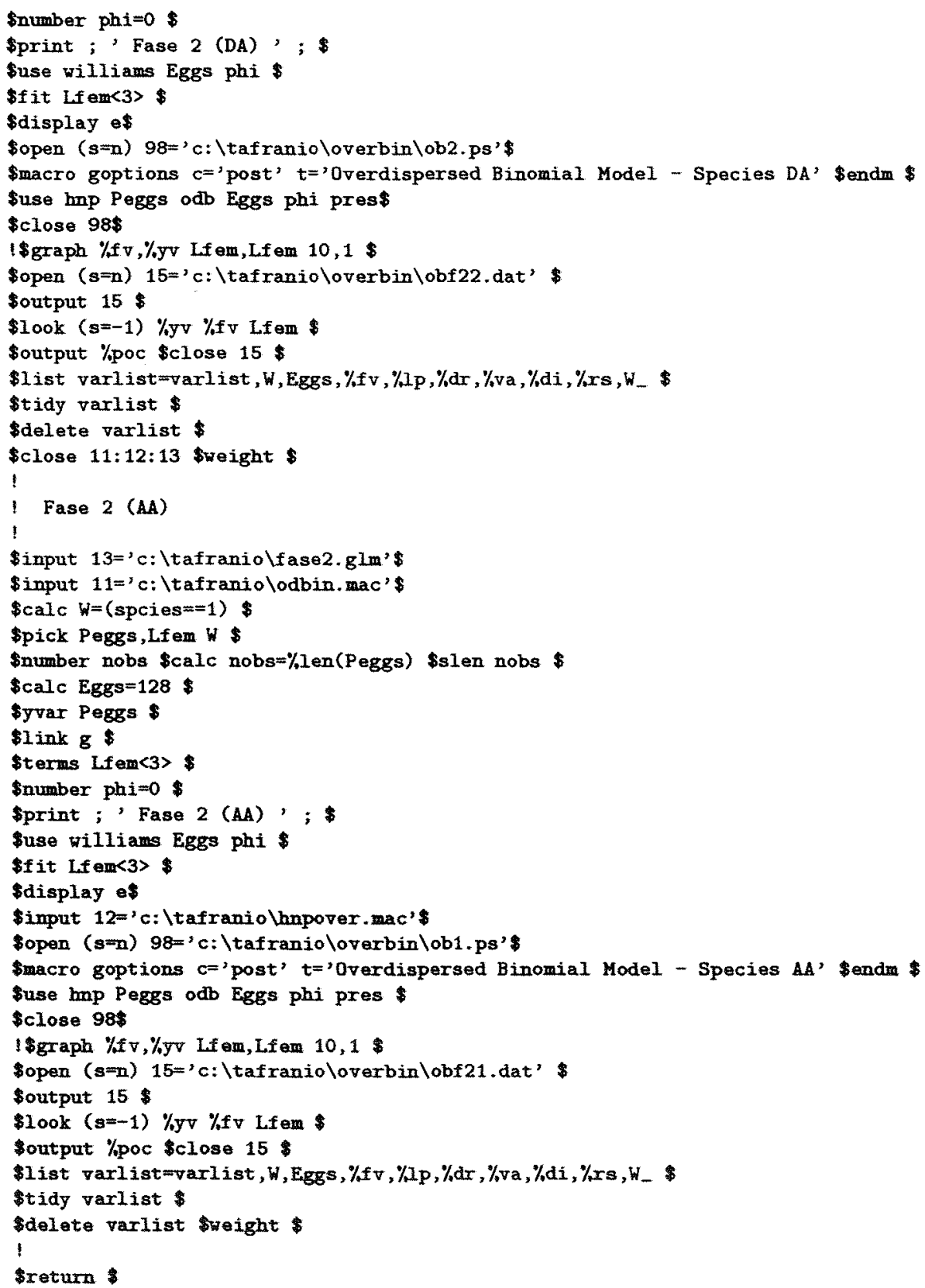


Fase 2 - modelo para linhagem 1

\$input 10 'c: \tafraniolfase2.glm'

\$calc Eggs $=128$

: $W=($ Spcies=1) $\$$

\$pick Peggs, Eggs, Lfem W

Snumber Nobs \$calc Nobs=\%len(Peggs) \$slenght Nobs \$

\$imput 11='c: \tafraniolmixotst.mac' \$

\$macro Model 1 Sendmac \$

\$macro Zmodel 1 Sendmac $\$$

\$print : 'MODELO PARA EXPERIMENTO FASE 2 - LIMHAGEA 1 (AA)' : \$

Suse HFACTOR Peggs Eggs

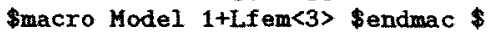

Suse HFACTOR Peggs Eggs \$

\$calc fitv=Znew*\% $\%$ : rs $=\%$ y - fitv $\$$

$\% \% \% \% \% \% \% \% \% \% \%$

$\$ c a l c$ rs=rs/\%sqrt (fitv*(1-fitv/eggs) $\$$

Sprint 'The estimated proportion of females that parasite is " $A(1)$

\$graph ( $s=1 \mathrm{t}=$ 'Grafico dos Residuos - Fase 2 (AA)' h='Valores Ajustados" $v=$ 'Residuos') rs fitv

$\$ g r a p h(s=1 \quad t=$ 'Valores observados e Ajustados - Fase 2 (AA)'

$\mathrm{h}=$ 'Log (numero de femeas)' $\nabla=$ 'numero de ovos parasitados')

$\%$ yv, fitv Lf em, Lfem $1,10 \$$

\$input $17=$ 'c: \tafranio hunpover.mac'

Simput 18='c: Itafranio hnpteste.mac' \$

\$calc phifix"phi : Afix"A

\$open $(s=n)$ 98='c: \tafranio $(m i x b o \backslash m b o 1 . p s ' \$$

Smacro goptions $c=$ 'post' $t=$ 'Overdispersed Mixture Model - Species $A A$ '

Sendmacro

Suse hnp Peggs mixbo Eggs * resid \$

close $98 \$$

\$open ( $s=n) 15=$ 'c: \tafraniolmixbo \mbof 21 dat' \$

Soutput 15

$\$ 1$ look $(s=-1) \% y v$ fitv Lfers

Soutput \%poc \$close 15

Isretura

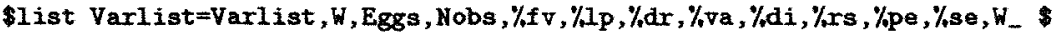

Stidy Varlist Maclist

\$delete Varlist Maclist

Sclose 17 : 18

\$weight

! Resetting the weights

Fase 2 modelo para Iinhagem 2

Sreinput $10 \$$

\$calc $W=($ Spcies=-2)

; Eggs=128

\$pick Peggs,Lfew,Eggs Peggs,Lfem,Eggs W

\$number Nobs Phi $=0$ \$calc Nobs=\%,len(Peggs) \$slenght Nobs

Sreinput 11

\$macro Hodel 1 Sendrac

\$macro Zmodel 1 Sendrac

Sprint : 'MODELO PARA EXPERTMENTO FASE 2 - LIMHAGEM 2 (DA)' :

luse HFACTOR Peggs Eggs

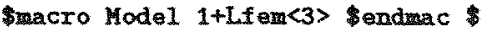

\$use HFACTOR Peggs Eggs

\$calc fitv-Znew $\%$ f

$1 \% \% \% \% \% \% \% \%$

\$calc rs $=r s / \%$ sqrt (fitv*(1-fitv/eggs)) $\$$

$\$ c a l c \mathrm{Zn}=(f i t \nabla>0.01)$.

print "The estimated proportion of females that parasite is , $A(1)$

Sgraph ( $s=1 t=$ 'Grafico dos Residuos - Fase 2 (DA)' h='Valores Ajustados' v'Residuos') rs fitv

Graph ( $s=1$ t='Valores obsexvados Ajustados - Fase 2 (DA)'

$\mathrm{h}=$ 'Log(numero de feaeas)' $\mathrm{L}=$ 'numero de ovos parasitados') 
$\% y v, f i t v / Z n$ Lfem,Lfem $1,10 \$$

\$input $17=$ 'c: : thafraniolhnpover.mac'

\$input 18='c: \tafranio lhnpteste.mac' \$

$\$ c a l c$ phifix=phi : Af $i x=A$

Sopen (s-n) 98=' c: Itafraniolmixbolmbo2.ps' \$

\$macro goptions $c=$ 'post' $t=$ 'Overdispersed Mixture Model - Species DA'

\$endmacro \$

\$use hnp Peggs mixbo Eggs * resid

Sclose $98 \$$

\$open $(s=n) 15=$ 'c: \tafraniolmizbolmof22, dat' \$

Soutput 15

\$look ( $s=-1) \%$ yv fitv Lfem $\$$

\$output \%poc \$close $15 \$$

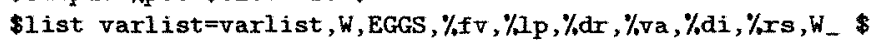

\$tidy Varlist Maclist

\$delete Varlist Maclist

Sweight

\$return

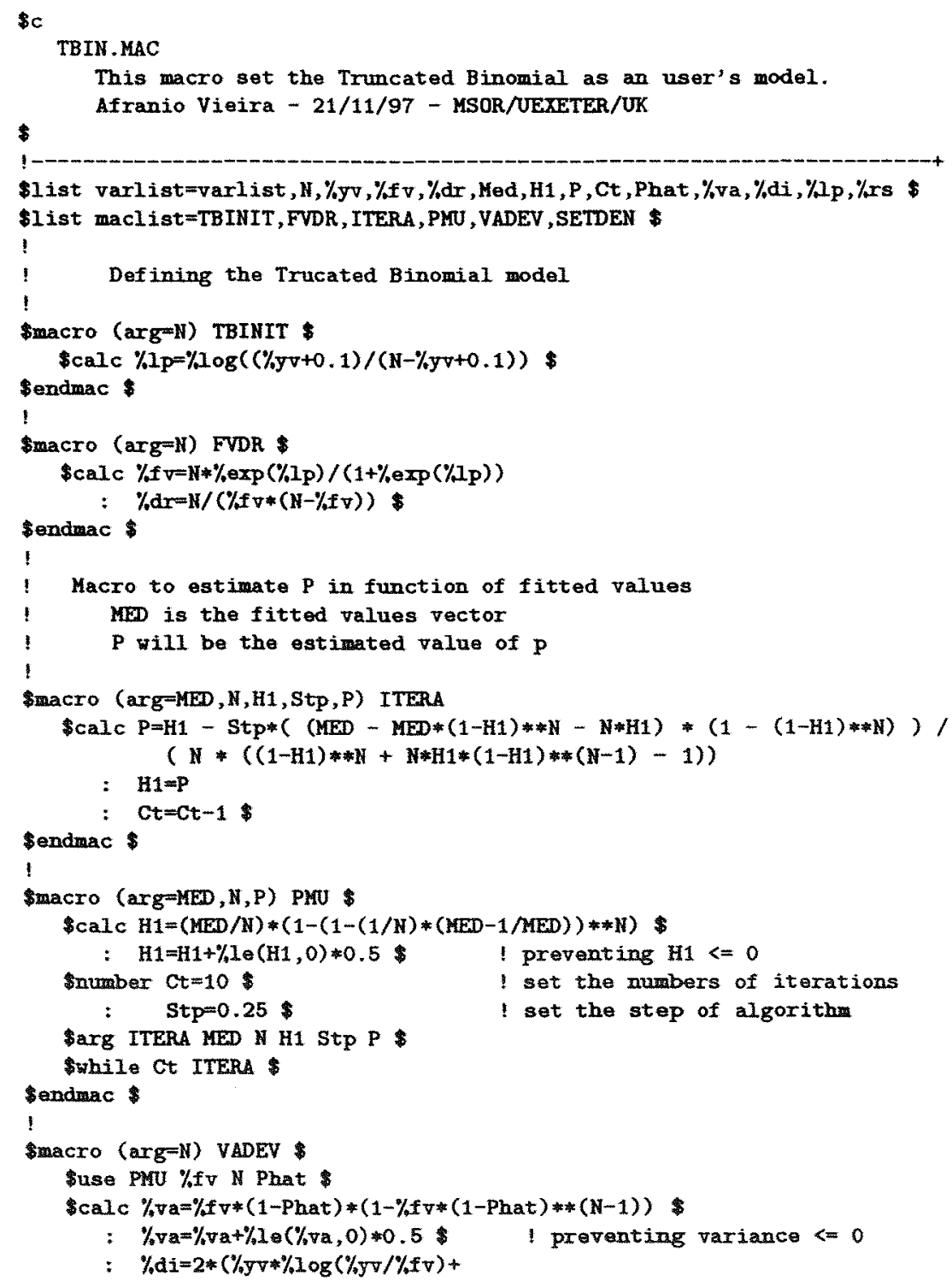




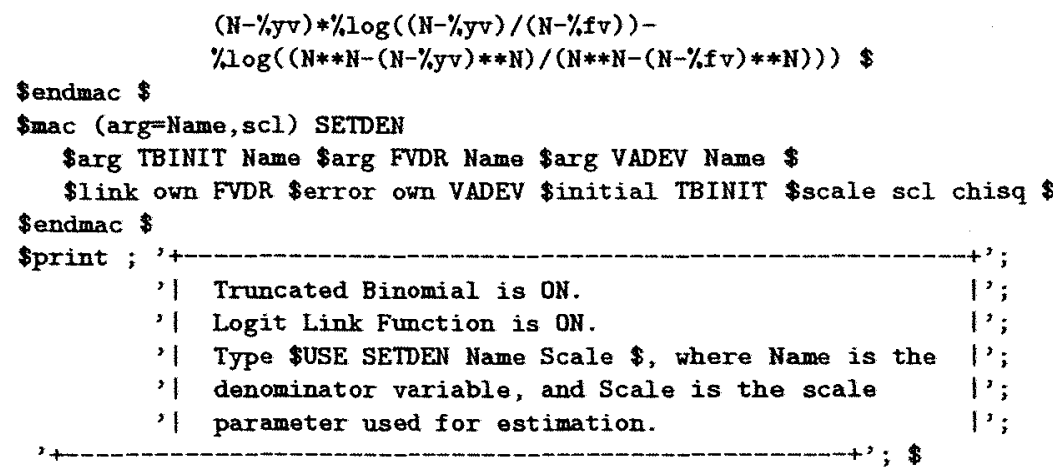

\$return \$

$\$ c$

Macro MIXMOD.MAC

Description: This macro fits a mixture model (Bernoulli-Binomial) thru the EM algorithm, to modelling binomial data inflated of zeros. This macro was based in Lambert (1992), Technometrics, v.34(1), p.1-14.

Afranio Vieira, 27/11/97 - MSOR/UEXETER/UK.

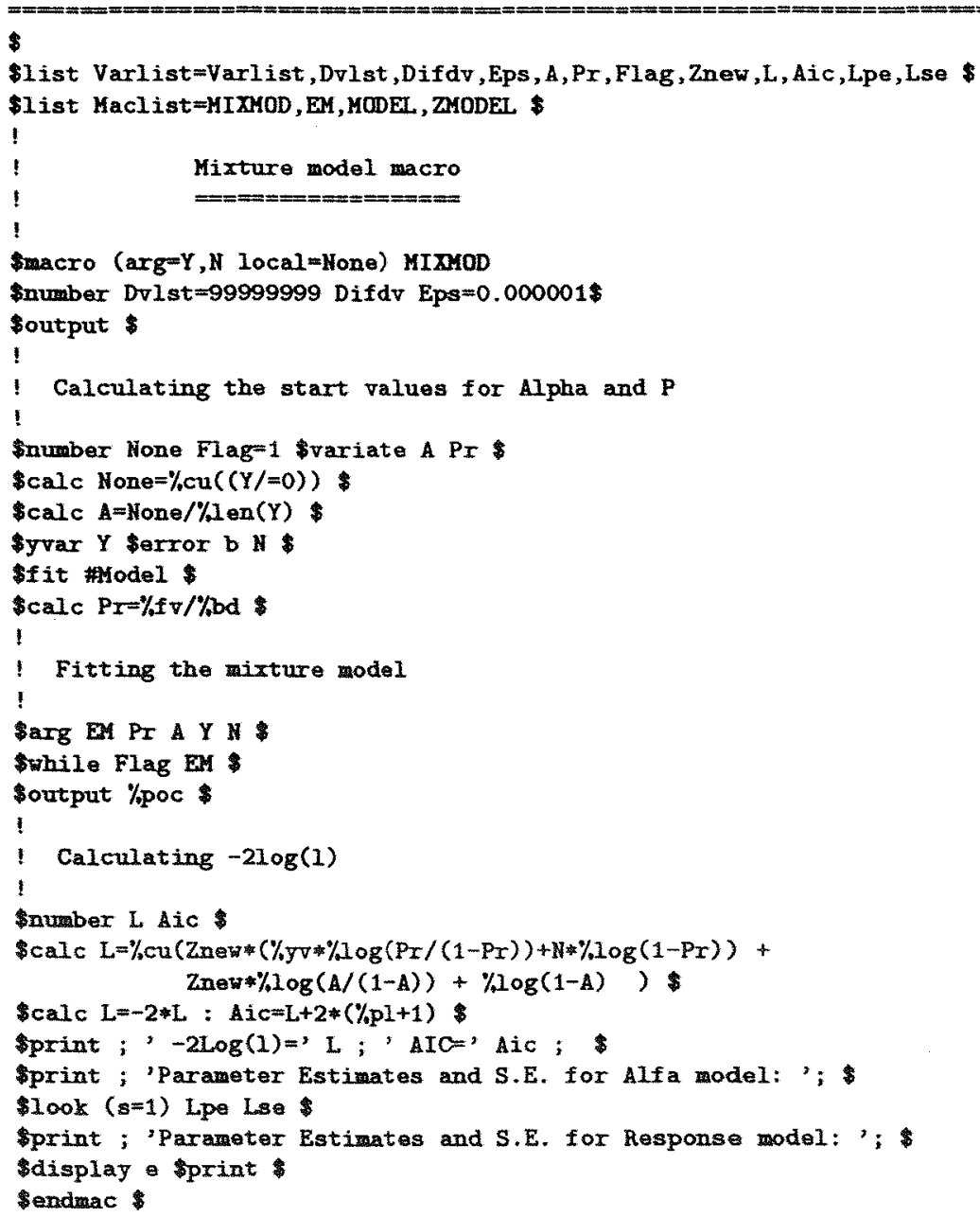




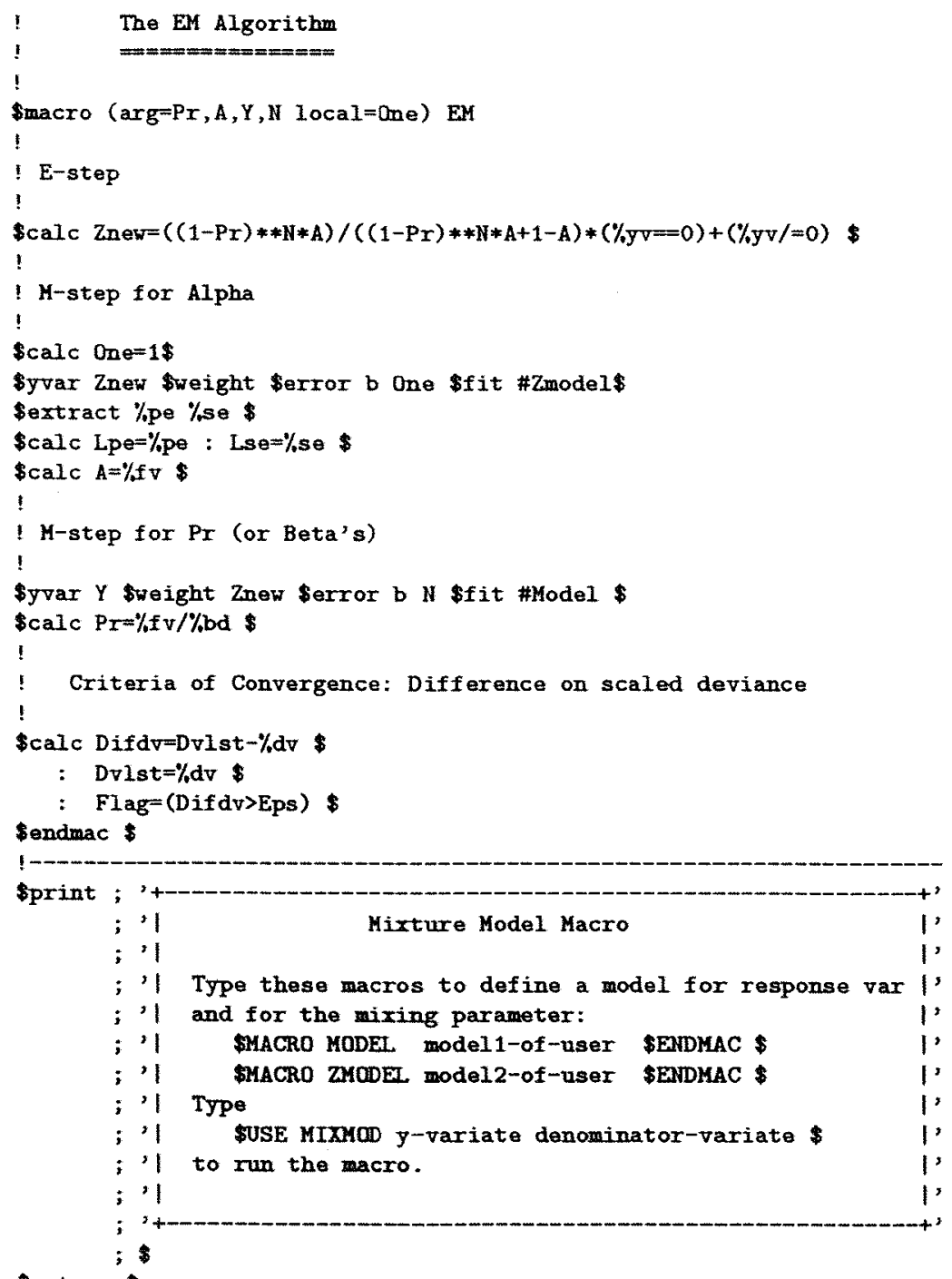

sreturn

$\$ c$

Macro HFACTOR (mixotst.ac)

Description: This macro fit a mixture model (Bernoulli-Overdispersed Bin)

thru the $E$ algorithm, using the heterogeneity factor model

in M-step.

This acro was developed to odelling Proportion data

inflated of zeros.

Afranio Vieira, 05/12/97 - MSOR/UEXETER/UK.

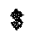

\$1ist Varlist=Varlist, Dvlst, Difdr, Eps, A, Pr,

Flag, Znew, L, Lpe, Lse, Phi, Abeta, Beta

list Maclist=HFACTOR, EM, HODEI, ZMODEL \$

!

1 Mixture model macro

1

Smacro (arg=Y,N local=None) HFACTOR

Snumber Drlst=99999999 Difdt Eps=0.000001

\$output 
$!$

Calculating the start values for Alpha and $P$

\$number None Flag=1 \$variate A Pr

\$calc None $=\%$ cu $((Y /=0))$ : A=None $/ \% \operatorname{len}(Y) \$$

\$yvar Y \$link g ferror b N \$scale 0 chis \$

$\$$ number Phi=0

$\$$ it \#todel \$calc Phi $=\%$ sc

Scalc Pr $=\%$ f $/ \%$ bd

Fitting the mixture model

\$variate Abeta Beta

Sarg Er Pr A Y Phi

\$vhile Flag EM \$

\$output \%poc \$

!

\$print ; 'Parameter Estimates for Alfa model: ';

$\$$ look $(s=1)$ Lpe $\$$

\$print ; 'Parameter Estimates and S.E. for Response model: ';

Sdisplay e \$

\$print; 'Estimate of $\mathrm{Phi}=$ ' Phi ; \$

$\$$ endmac

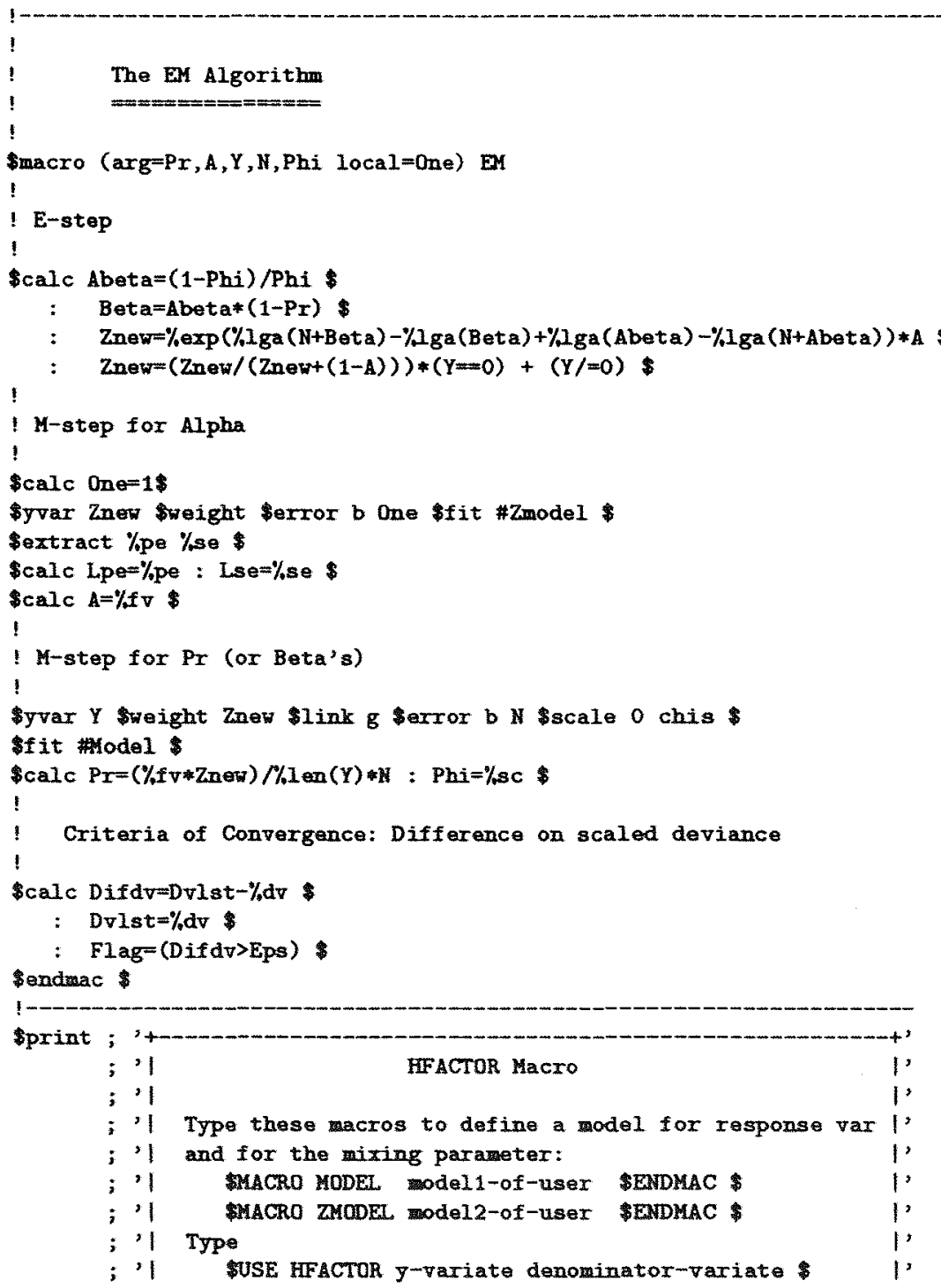



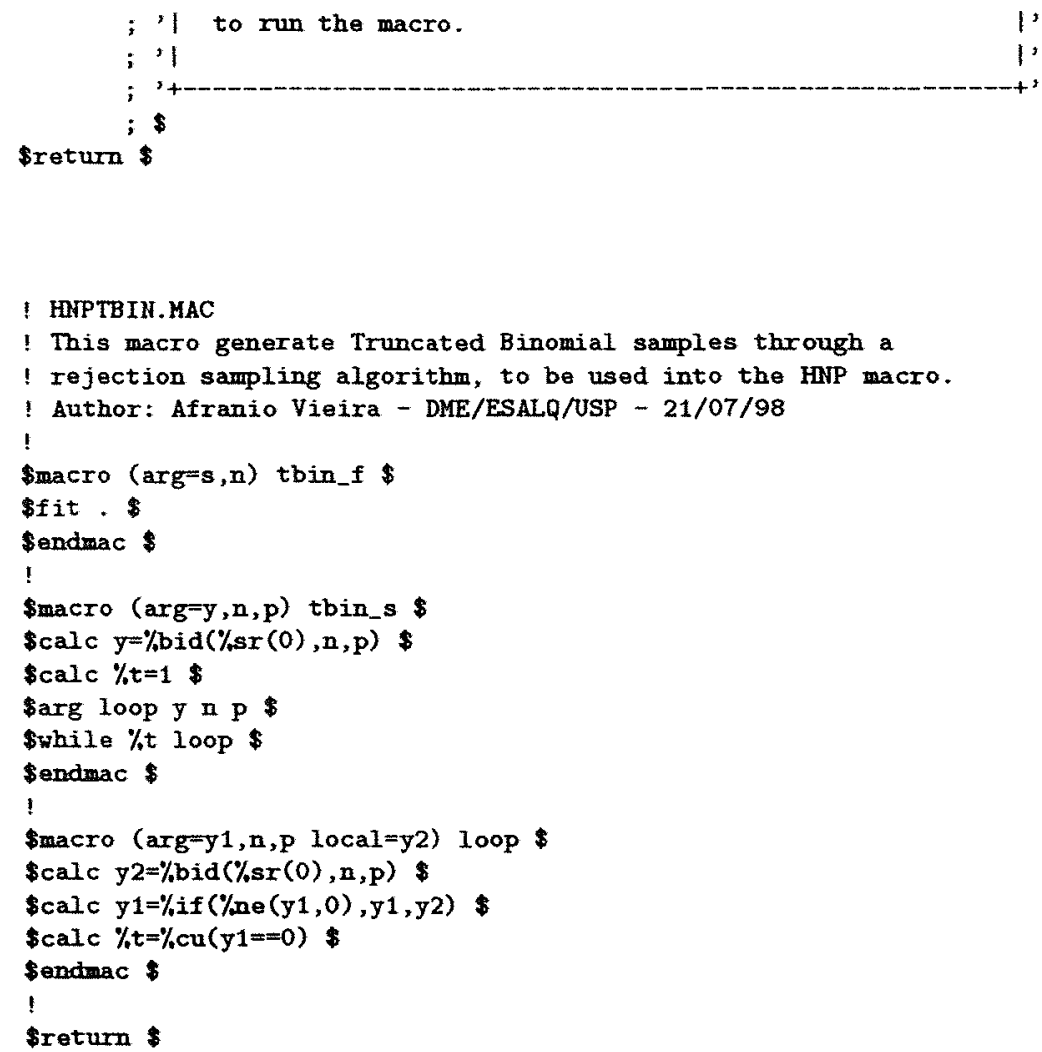


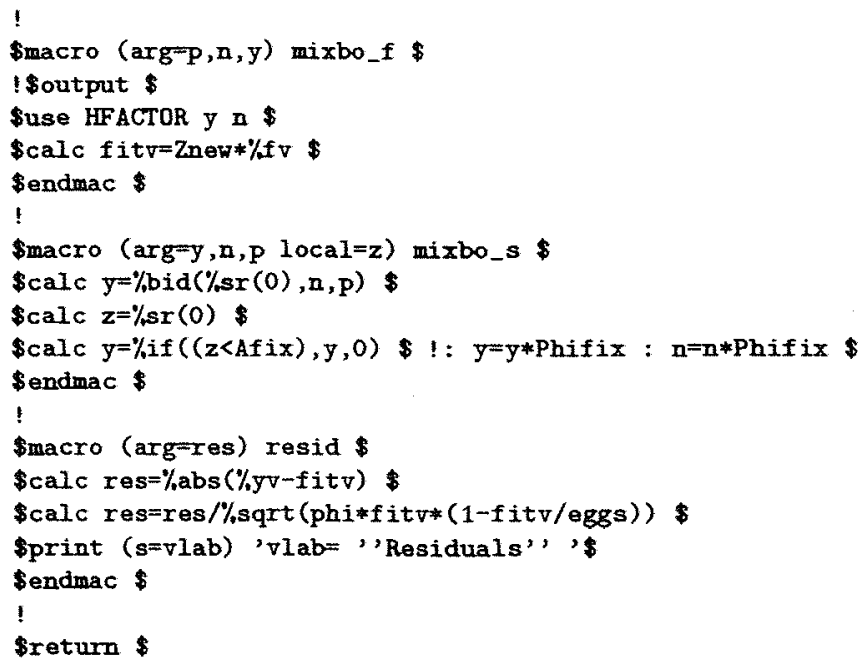




\section{APENNDICE 2 \\ Conjunto de Dados}


\$macro IAFO

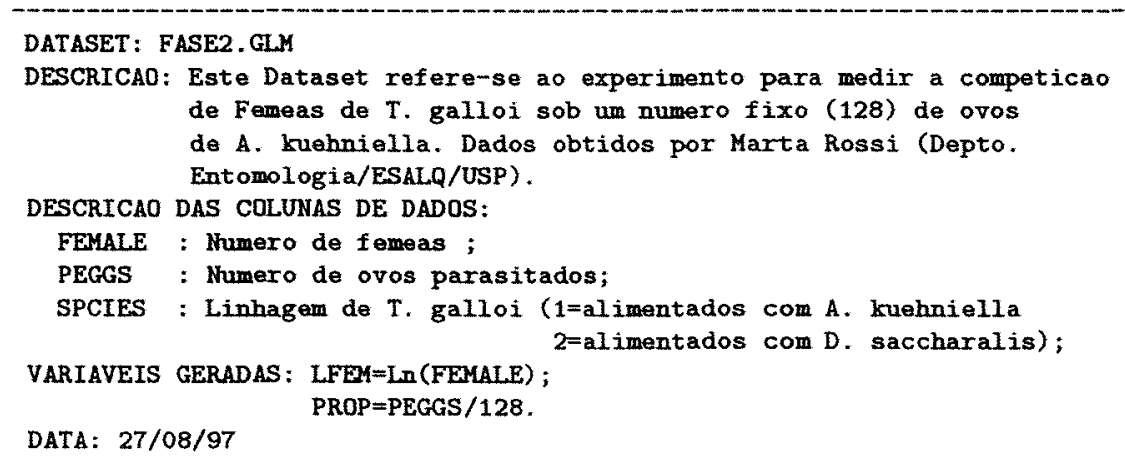

\$endmacro

\$slenght $140 \$$

$\$ 1$ ist VARLIST=FEMALE, PEGGS, SPCIES, LFEM, PROP \$

\$data X1 X2 FEMALE PEGGS SPCIES \$

Sread

\begin{tabular}{|c|c|c|c|c|}
\hline 18 & 128 & 2 & 40 & 1 \\
\hline 18 & 128 & 2 & 73 & 1 \\
\hline 18 & 128 & 2 & 32 & 1 \\
\hline 18 & 128 & 2 & 30 & 1 \\
\hline 18 & 128 & 2 & 38 & 1 \\
\hline 18 & 128 & 2 & 80 & 1 \\
\hline 18 & 128 & 2 & 40 & 1 \\
\hline 18 & 128 & 2 & 39 & 1 \\
\hline 18 & 128 & 2 & 44 & 1 \\
\hline 18 & 128 & 2 & 52 & 1 \\
\hline 19 & 128 & 4 & 70 & 1 \\
\hline 19 & 128 & 4 & 71 & 1 \\
\hline 19 & 128 & 4 & 35 & 1 \\
\hline 19 & 128 & 4 & 122 & 1 \\
\hline 19 & 128 & 4 & 73 & 1 \\
\hline 19 & 128 & 4 & 72 & 1 \\
\hline 19 & 128 & 4 & 71 & 1 \\
\hline 19 & 128 & 4 & 100 & 1 \\
\hline 19 & 128 & 4 & 34 & 1 \\
\hline 19 & 128 & 4 & 32 & 1 \\
\hline 20 & 128 & 8 & 112 & 1 \\
\hline 20 & 128 & 8 & 122 & 1 \\
\hline 20 & 128 & 8 & 99 & 1 \\
\hline 20 & 128 & 8 & 74 & 1 \\
\hline 20 & 128 & 8 & 63 & 1 \\
\hline 20 & 128 & 8 & 125 & 1 \\
\hline 20 & 128 & 8 & 48 & 1 \\
\hline 20 & 128 & 8 & 96 & 1 \\
\hline 20 & 128 & 8 & 64 & 1 \\
\hline 20 & 128 & 8 & 96 & 1 \\
\hline 21 & 128 & 16 & 128 & 1 \\
\hline 21 & 128 & 16 & 128 & 1 \\
\hline 21 & 128 & 16 & 128 & 1 \\
\hline 21 & 128 & 16 & 127 & 1 \\
\hline 21 & 128 & 16 & 109 & 1 \\
\hline 21 & 128 & 16 & 121 & 1 \\
\hline 21 & 128 & 16 & 128 & 1 \\
\hline 21 & 128 & 16 & 119 & 1 \\
\hline 21 & 128 & 16 & 125 & 1 \\
\hline 21 & 128 & 16 & 98 & 1 \\
\hline 22 & 128 & 32 & 115 & 1 \\
\hline 22 & 128 & 32 & 118 & 1 \\
\hline 22 & 128 & 32 & 128 & 1 \\
\hline 22 & 128 & 32 & 128 & 1 \\
\hline 22 & 128 & 32 & 120 & 1 \\
\hline
\end{tabular}




\begin{tabular}{|c|c|c|c|c|}
\hline 22 & 128 & 32 & 111 & 1 \\
\hline 22 & 128 & 32 & 123 & 1 \\
\hline 22 & 128 & 32 & 116 & 1 \\
\hline 22 & 128 & 32 & 121 & 1 \\
\hline 22 & 128 & 32 & 113 & 1 \\
\hline 23 & 128 & 64 & 118 & 1 \\
\hline 23 & 128 & 64 & 128 & 1 \\
\hline 23 & 128 & 64 & 105 & 1 \\
\hline 23 & 128 & 64 & 113 & 1 \\
\hline 23 & 128 & 64 & 127 & 1 \\
\hline 23 & 128 & 64 & 106 & 1 \\
\hline 23 & 128 & 64 & 112 & 1 \\
\hline 23 & 128 & 64 & 126 & 1 \\
\hline 23 & 128 & 64 & 110 & 1 \\
\hline 23 & 128 & 64 & 110 & 1 \\
\hline 24 & 128 & 128 & 110 & 1 \\
\hline 24 & 128 & 128 & 108 & 1 \\
\hline 24 & 128 & 128 & 100 & 1 \\
\hline 24 & 128 & 128 & 105 & 1 \\
\hline 24 & 128 & 128 & 120 & 1 \\
\hline 24 & 128 & 128 & 101 & 1 \\
\hline 24 & 128 & 128 & 103 & 1 \\
\hline 24 & 128 & 128 & 102 & 1 \\
\hline 24 & 128 & 128 & 98 & 1 \\
\hline 24 & 128 & 128 & 95 & 1 \\
\hline 26 & 128 & 2 & 0 & 2 \\
\hline 26 & 128 & 2 & 0 & 2 \\
\hline 26 & 128 & 2 & 0 & 2 \\
\hline 26 & 128 & 2 & 0 & 2 \\
\hline 26 & 128 & 2 & 0 & 2 \\
\hline 26 & 128 & 2 & 50 & 2 \\
\hline 26 & 128 & 2 & 65 & 2 \\
\hline 26 & 128 & 2 & 30 & 2 \\
\hline 26 & 128 & 2 & 62 & 2 \\
\hline 26 & 128 & 2 & 48 & 2 \\
\hline 27 & 128 & 4 & 0 & 2 \\
\hline 27 & 128 & 4 & 0 & 2 \\
\hline 27 & 128 & 4 & 0 & 2 \\
\hline 27 & 128 & 4 & 0 & 2 \\
\hline 27 & 128 & 4 & 0 & 2 \\
\hline 27 & 128 & 4 & 0 & 2 \\
\hline 27 & 128 & 4 & 0 & 2 \\
\hline 27 & 128 & 4 & 62 & 2 \\
\hline 27 & 128 & 4 & 3 & 2 \\
\hline 27 & 128 & 4 & 1 & 2 \\
\hline 28 & 128 & 8 & 0 & 2 \\
\hline 28 & 128 & 8 & 0 & 2 \\
\hline 28 & 128 & 8 & 0 & 2 \\
\hline 28 & 128 & 8 & 0 & 2 \\
\hline 28 & 128 & 8 & 0 & 2 \\
\hline 28 & 128 & 8 & 49 & 2 \\
\hline 28 & 128 & 8 & 19 & 2 \\
\hline 28 & 128 & 8 & 22 & 2 \\
\hline 28 & 128 & 8 & 51 & 2 \\
\hline 28 & 128 & 8 & 65 & 2 \\
\hline 29 & 128 & 16 & 0 & 2 \\
\hline 29 & 128 & 16 & 0 & 2 \\
\hline 29 & 128 & 16 & 0 & 2 \\
\hline 29 & 128 & 16 & 0 & 2 \\
\hline 29 & 128 & 16 & 0 & 2 \\
\hline 29 & 128 & 16 & 57 & 2 \\
\hline 29 & 128 & 16 & 35 & 2 \\
\hline 29 & 128 & 16 & 52 & 2 \\
\hline 29 & 128 & 16 & 37 & 2 \\
\hline 29 & 128 & 16 & 58 & 2 \\
\hline 30 & 128 & 32 & 0 & 2 \\
\hline 30 & 128 & 32 & 0 & 2 \\
\hline
\end{tabular}




$\begin{array}{ll}30 & 128 \\ 30 & 128 \\ 30 & 128 \\ 30 & 128 \\ 30 & 128 \\ 30 & 128 \\ 30 & 128 \\ 30 & 128 \\ 31 & 128 \\ 31 & 128 \\ 31 & 128 \\ 31 & 128 \\ 31 & 128 \\ 31 & 128 \\ 31 & 128 \\ 31 & 128 \\ 31 & 128 \\ 31 & 128 \\ 32 & 128 \\ 32 & 128 \\ 32 & 128 \\ 32 & 128 \\ 32 & 128 \\ 32 & 128 \\ 32 & 128 \\ 32 & 128 \\ 32 & 128 \\ 32 & 128\end{array}$

32
32
32
32
32
32
32
32
64
64
64
64
64
64
64
64
64
64
128
128
128
128
128
128
128
128
128
128

2

$\begin{array}{llll}128 & 32 & 0 & 2\end{array}$

$\begin{array}{llll}128 & 32 & 0 & 2\end{array}$

$\begin{array}{lll}32 & 120 & 2\end{array}$

$\begin{array}{llll}28 & 64 & 0 & 2\end{array}$

$\begin{array}{llll}64 & 0 & 2\end{array}$

$64 \quad 002$

$64 \quad 004$

$\begin{array}{lll}64 & 57 & 2\end{array}$

$\begin{array}{lll}64 & 77 & 2\end{array}$

$\begin{array}{llll}28 & 128 & 99 & 2 \\ 128 & 0 & 0\end{array}$

$\begin{array}{llll}28 & 128 & 0 & 2\end{array}$

$128 \quad 0 \quad 2$

2

$38 \quad 2$

$21-2$

$82 \quad 2$

$42 \quad 2$

$90 \quad 2$

812

$\$$ delete $X_{1} X_{2} \$$

\$factor SPCIES $2 \$$

$\$$ calc LFEM=\%log (FEMALE)

: PROP=PEGGS/128

\$return 